\title{
Zur sprachlichen Kreativität Alma M. Karlins - nachgewiesen an Phraseolexemen und satzwertigen Phraseologismen in ,Windlichter des Todes. Roman aus Siam‘ (1933)
}

\author{
Inge POHL
}

\begin{abstract}
Aspects of linguistic creativity in the work of Alma M. Karlin - demonstrated using phraseolexemes and sentence-like phraseologisms in 'Windlichter des Todes. Roman aus Siam' (1933)

Phraseolexemes and sentence-like phraseologisms taken from the novel 'Windlichter des Todes. Roman aus Siam' (1933) prove that Alma M. Karlin is rightly known as a linguistically creative author. It can be shown that Karlin often uses patterns of occasionalisms (modifications and neologisms) to support the aesthetic and artistic value of the novel.
\end{abstract}

Keywords: Alma M. Karlin, linguistic creativity, phraseolexemes, proverbs and familiar quotations, phraseological functions in literary texts, phraseological isotopy

DOI: doi.org/10.15452/StudiaGermanistica.2020.27.0004

\section{Textgrundlage und Fragestellungen}

Zur sprachlichen Kreativität Alma M. Karlins, der ausschließlich in Deutsch schreibenden slowenischen Schriftstellerin, sind in jüngerer Zeit einzelne Aufsätze erschienen, die sich textlinguistisch-stilistisch mit formalen, semantischen und funktionalen Aspekten der von ihr gebrauchten sprachlichen Einheiten befassen, wie z. B. zu Personenbenennungen (vgl. Ehrhardt 2015), Euphemismen und Dysphemismen (vgl. Štavbar 2019), Okkasionalismen (vgl. Pohl 2019b). Noch unterrepräsentiert ist die linguistisch-stilistische Analyse von Phraseologismen bei Karlin, vgl. jedoch Wolff/Ehrhardt (2017) zum Vergleich von reise- und kinderliterarischen Texten. Mir geht es um die Untersuchung des Gebrauchs von ausgewählten Phraseologismen in einem einzelnen belletristischen Werk Karlins, das unter diesem Aspekt bislang nicht untersucht wurde. Fokussiert ist hier die bereits in Pohl (2019a, b, c) festgestellte sprachliche Kreativität Alma M. Karlins, die ich auch für den Phraseologismengebrauch im ausgewählten belletristischen Werk Karlins annehme.

Der Roman ,Windlichter des Todes. Roman aus Siam“ erschien 1933, die Handlung spielt in Bangkok, der damaligen Hauptstadt Siams (heute Thailand). Erzählt werden von einem auktorialen Erzähler (nachfolgend aE) die Schicksale von sieben Frauen, die im interkulturellen Bangkok um 
1930 Erfahrungen mit männlichen Partnern suchen, bis sich unterschiedliche Beziehungslösungen ergeben. Als grundlegende Textbedeutung ist das Selbstverständnis von Frauen und das Verhältnis von Mann und Frau in interkultureller Perspektive zu sehen (vgl. Pohl 2019a:142 f.). Wie in einem Liebesroman geht es um geglückte vs. gescheiterte Beziehungen. Die Textbotschaft fokussiert allerdings eine ethisch-moralische Auffassung Karlins im Spannungsfeld der Diskurse „Liebe zwischen Mann und Frau“ und „Rassereinheit vs. Rassenmischung“: Karlin skaliert Rassereinheit als höchst positiven Wert, den sie zur Legitimation von Paarbeziehungen im interkulturellen Raum Siam heranzieht. Die Romanhandlung entspricht Karlins These: Nur reinrassige Verbindungen glücken (Primula ${ }^{1}$ geht eine Liebesheirat mit Dr. Nathfield ein, Emma wird die Geliebte des Multimillionärs Ehrental, die siamesischen Prinzessin Bhin heiratet traditionsgemäß einen siamesischen Prinzen). In gemischtrassigen Paarbeziehungen degradieren sich die Mischlingsfrauen (Anna gibt ihre Religion auf und wird Zweitfrau eines Mohammedaners), sterben gar (wie Klementine) oder bleiben ehe- und kinderlos (wie das ältere Fräulein d'Albuquerque) (vgl. Pohl 2019a).

Die Lektüre des Untersuchungsromans erhellt, dass Phraseologismen rein quantitativ ein relevantes Stilelement darstellen, insbesondere Komparative Phraseologismen, z. B. die Brüste der Eurasierin wuchsen wie Wellen bei losbrechendem Sturm (WT 24), ${ }^{2}$ Phraseolexeme, z. B. daß sie bis zum Hals in Schulden steckt (WT 86), satzwertige Phraseologismen, z. B. Hier liegt der Hase im Pfeffer. (WT 134), Phraseologische Wortpaare und Nominationsstereotype, z. B. Kind auf Kind hatte sie gedankenlos auf den Strom des Lebens geworfen (WT 62), Kommunikative Formeln, z. B. Bei des Teufels Urgroßmutter! (WT 67) und Phraseoschablonen, z. B. der Zug rollte und rollte (WT 145). Bei einer Romanlänge von 177 Seiten verteilen sich die von mir ermittelten ca. 160 Komparativen Phraseologismen, 37 satzwertigen Phraseologismen, 36 Phraseolexeme, 14 Nominationsstereotype, 10 Phraseologischen Wortpaare, 6 Kommunikativen Formeln, 5 Phraseoschablonen (insgesamt 268) dergestalt, dass pro Seite 1,5 Phraseologismen von der Romanautorin eingesetzt sind. ${ }^{3}$ Damit befindet sich die Phraseologismusdichte im Roman in einem mittleren Bereich (vgl. Richter-Vapaatalo 2007). Für die nachfolgende textlinguistisch-stilistische Untersuchung konzentriere ich mich auf Phraseolexeme und satzwertige Phraseologismen. Mein Untersuchungskorpus besteht aus 73 aufgrund meiner Sprachkompetenz ${ }^{4}$ ausgewählten Belegen. In der Darstellung kennzeichne ich alle Sprachbeispiele kursiv, einen Phraseologismus in seiner Grundform $^{5}$ zusätzlich fett, Modifikationen innerhalb eines Phraseologismus sind unterstrichen. Kursivierungen außerhalb des Phraseologismus verweisen auf andere Textteile mit semantischem Bezug zum Phraseologismus. Die Zitation der Phraseologismen erfolgt im Wesentlichen mit dem Umgebungssatz.

Zum Begriff der sprachlichen Kreativität existiert noch keine allgemein anerkannte Definition (vgl. Brodbeck 2006). Exemplarisch aus dem linguistischen Bereich sind die Arbeiten von Musolff (1990), Polzin (1997) und Pusch (1997) zu nennen. Sprachliche Kreativität als komplexes Phänomen ist in meinem Zusammenhang auf objektiv vorhandene Sprachprodukte, die Phraseologismen, und die sprachlich-kreative Handlung der Autorin zu beziehen. Letztere kann lediglich hypothetisch und subjektiv in ihrer Originalität, Unüblichkeit, Ungewöhnlichkeit und Neuartigkeit des SpracheGebrauchens hinsichtlich des ästhetisch-künstlerischen Anspruchs des Untersuchungsromans abgesteckt werden. Die Formung kreativer Sprachprodukte generell erfolgt im Rahmen der geläufigen, usualisierten Muster, überschreitet jedoch deren normierte Grenzen und scheint im Widerspruch

Die literarischen Figuren führe ich hier bis auf wenige Ausnahmen lediglich mit Vor- oder Familiennamen auf.

WT $=$,Windlichter des Todes. Roman aus Siam‘, es folgt die Seitenzahl des Belegs. In eckigen Klammern gebe ich die Autorin/den Autor des Phraseologismus sowie einen denotativen oder kompositorischen Bezug an.

Diese Zahlen besitzen keine absolute Wertigkeit, da lediglich meine muttersprachliche Sprachkompetenz das Auswahlkriterium darstellt, wobei ich mir der Subjektivität dieses Kriteriums bewusst bin. Das Materialkorpus ist jedoch ausreichend groß, so dass die Ergebnisse tendenziell als repräsentativ gelten können.

4 Zur Überprüfung wurden die im Literaturverzeichnis aufgeführten Wörterbücher herangezogen.

Unter Grundform des Phraseologismus verstehe ich mit Richter-Vapaatalo (2007:83) „die kontextuell ,gefüllte Nennform““, d. h. den Phraseologismus im Beleg mit all seinen Aktanten. 
zur Einhaltung von konventionalisierten Regeln im Sprachgebrauch zu stehen. Den Phraseologismengebrauch im Karlinschen Roman bewerte ich aufgrund meiner Erkenntnisse als kreativ. Karlin nutzt die latent kreativen Potenzen der Phraseologismen sprachbewusst aus, die begründet sind in der Mehrwortstruktur, in der nur relativen Stabilität und in den metaphorischen Prozessen, die zur Idiomatizität führen. Außerdem lässt sich mit der potentiellen freien und der idiomatischen Lesart von Phraseologismen kreativ spielen (vgl. Palm 1995:62). Der Kreativitätsbegriff ist hier situativ, bezogen auf ein literarisches Werk mit seiner Autorin, auf Sprachprodukte anwendbar, die „neu“ und „wertvoll“ sind, wobei die Relativität der Kriterien zu beachten ist: Was als „neu“ bewertet wird, kann vom jeweiligen Betrachterstandpunkt abhängig sein. Der Begriff „Wert“ enthält historische, persönliche, ethische usw. Aspekte, ist also nicht sinnvoll einzugrenzen (vgl. ausführlich Brodbeck 2006).

Um meine Hypothese zur sprachlichen Kreativität Karlins bezüglich der Phraseologismen zu verifizieren, gehe ich folgenden Fragen nach: (i) Unter formalem Aspekt: Welche Arten von Phraseologismen gebraucht Karlin? Kreiert die Autorin okkasionelle ${ }^{6}$ und/oder Autorphraseologismen? ${ }^{7}$ Welche Arten von Modifikationen nutzt Karlin? Wie ist das Verhältnis von modifizierten Phraseologismen zu Phraseologismen, die sprachsystemgetreu gebraucht werden? Wie werden Phraseologismen in den Kontext eingebunden, z. B. durch Kommentierungen bzw. Modifikationen? (ii) Unter semantischem Aspekt: Welche Konzepte liegen den Phraseologismen zugrunde? Gibt es semantische Vernetzungen zwischen den Phraseologismen? Welcher semantische Mehrwert wird durch Modifikationen erbracht? Wie nutzt Karlin Metaphorisierungen bei okkasionellen Phraseologismen aus? (iii) Unter funktionalem Aspekt: Von welchen Wirkungen der Phraseologismen (inklusive ihrer Konzepte) auf potentielle Leser lässt sich hinsichtlich der Autorintention, der Textstrukturierung und der Figurencharakterisierung hypothetisch ausgehen?

\section{Begrifflichkeiten}

Unter „Phraseologie“ fasst man heute allgemein zwei Bedeutungsvarianten, zum einen die sprachwissenschaftliche Teildisziplin, die sich mit der Erforschung von Phraseologismen beschäftigt, und zum anderen das Inventar von Phraseologismen in einer bestimmten Einzelsprache (vgl. Fleischer 1997:3). In meiner Untersuchung, die sich auf den phraseologischen Bestand in einem literarischen Werk orientiert, verwende ich den Begriff „Phraseologismus“ im allgemeinsten Sinne: Ich verstehe darunter ,eine Verbindung von zwei oder mehr Wörtern [...], wenn (1) die Wörter eine durch die syntaktischen und semantischen Regularitäten der Verknüpfung nicht voll erklärbare Einheit bilden, und wenn (2) die Wortverbindung in der Sprachgemeinschaft [...] gebräuchlich ist" (Handbuch 1982:1). In der linguistischen Diskussion besteht Konsens darüber, dass bei der Klassifizierung von Phraseologismen Zentrum und Peripherie anzunehmen sind. Unterschiedlich sind die Meinungen darin, welche Arten von Phraseologismen die jeweiligen Bereiche konstituieren. Ich orientiere mich an Wotjak (1992), Palm (1995) und Fleischer (1997).

Phraseolexeme bilden nach Auffassung der Mehrzahl von Linguisten das Zentrum des phraseologischen Bestandes, sie besitzen mindestens ein Autosemantikon, ,sind kommunikativ-grammatisch (nach Tempus, Person, Modus die Verben, nach Kasus die substantivischen Phraseolexeme) mehr oder weniger variabel (innerhalb der erörterten Beschränkungen, aber doch ohne stabile Satzstruktur wie die festgeprägten Sätze [...])“ (Fleischer 1997:68). Phraseolexeme besitzen die

\footnotetext{
Von okkasionellen Phraseologismen spricht man, wenn „ein charakteristisches phraseologisierbares Strukturmodell (Wortpaare, angeschlossene Vergleichskonstruktionen) individuell mit ungewöhnlichem lexikalischen Material ,gefüllt"“wird (Fleischer 1997:65).

7 Autorphraseologismen sind feste Verbindungen, die nur innerhalb eines literarischen Werkes vom Autor aufgebaut und mit Sinn gefüllt werden (vgl. Burger 2007:49).
} 
phraseologischen Hauptmerkmale der Polylexikalität, ${ }^{8}$ der Idiomatizität, ${ }^{9}$ der Stabilität/Festigkeit ${ }^{10}$ und der Lexikalisierung ${ }^{11}$ (und somit Reproduzierbarkeit). ${ }^{12}$ Sie können vollidiomatisch (jmdm. einen Stempel aufdrücken; WT 44) oder teilidiomatisch (es regnet Spitzbuben; WT 48) sein.

Insofern ein oder zwei der genannten Hauptmerkmale fehlen, rücken die Phraseologismen in den peripheren Bereich. Zur Peripherie zählt man (vgl. Fleischer 1997:69 ff.) potentielle oder individuelle Phraseolexeme, Nominationsstereotype, Kommunikative Formeln, Phraseoschablonen, Funktionsverbgefüge. Als besondere Strukturtypen von Phraseologismen werden genannt die Komparativen Phraseologismen und die Phraseologischen Wortpaare. Eine wesentliche Frage in der bisherigen Fachdiskussion ist darauf gerichtet, ob Sprichwörter, Geflügelte Worte, formelhafte Texte zu den Gegenständen der Phraseologie gehören. Ich folge einer weiten Phraseologie-Auffassung, die beispielsweise Palm (1995), Stein (1995) oder Stumpf (2015) eingebracht haben.

Hinsichtlich meiner Fragestellung, inwieweit Karlin Phraseologismen kreativ gebraucht, sind die Hauptmerkmale von Phraseologismen, das Kategorienpaar Stabilität und Variabilität, in den Blick zu nehmen. Im Verständnis des Handbuchs (1982:67 f.) hat sich eingebürgert, „Zwischen usuellen, lexikographisch allerdings nicht immer konsequent kodifizierten Varianten (Variation) und okkasionellen, textgebundenen Modifikationen (Modifikation)“ (Fleischer 1997:263) zu unterscheiden. Da Karlin kaum Varianten einsetzt, befasse ich mich ausführlicher mit der Modifikation, wozu in der Fachliteratur verschiedene Begrifflichkeiten auffindbar sind. Einigkeit besteht darin, dass Modifikation die okkasionellen morphologisch-syntaktischen oder lexikalischen Veränderungen innerhalb eines Textes anzeigt (vgl. Richter-Vapaatalo 2007:46). Für die im Untersuchungsroman auftretenden Modifikationen nutze ich eine weite Auslegung des Begriffs im Sinne des Handbuchs (1982:70 ff.; ebenso Wotjak 1992:153 ff.; Palm 1995:71 ff.), welches wendungsexterne phraseologismusrelevante Veränderungen mit einbezieht. Folglich unterscheide ich bei (i) phraseologismusinternen Modifikationen semantische Modifikationen (lexikalische Substitution, Expansion, Wechsel von Affirmation und Negation; Erklärungen hierzu in den Kapiteln 3 und 4) und strukturelle Modifikationen (Reduktion, Koordinierung, ${ }^{13}$ Abtrennung, ${ }^{14}$ Nominalisierung u. a.). Zu (ii) phraseologismusrelevanten externen (kontextualen) Bezugnahmen zähle ich z. B. Häufung, Kontamination mit eventuellen Katachresen, ${ }^{15}$ Verweise im Kontext, ${ }^{16}$ Verletzung semantischer Selektionsbedingungen, ${ }^{17}$ sprachliche und metasprachliche Kommentierung inkl. Verwendung von

$8 \quad$ Ein Phraseologismus besteht aus mehr als einem Wort. Die Obergrenze phraseologischer Einheiten wird noch diskutiert (vgl. Obergrenze Satz bei Burger 2007:15; Obergrenze formelhafte Texte bei Stein 1995).

9 Von idiomatischer Bedeutung spricht man, wenn „die Komponenten eine durch die syntaktischen und semantischen Regularitäten der Verknüpfung nicht voll erklärbare Einheit bilden“ (Burger 2007:15).

10 Unter Stabilität wurde bislang vorrangig die strukturelle Festigkeit verstanden. In neuerer Literatur werden pragmatische (Geläufigkeit in einem ,synchronen Sprachquerschnitt“) und psycholinguistische Aspekte (vgl. Reproduzierbarkeit) in das Kriterium der Stabilität/Festigkeit einbezogen (vgl. Burger 2007:16 f.). Das Merkmal der Stabilität/Festigkeit ist relativ, vgl. das Vorhandensein optionaler Variation und Modifikation.

11 Lexikalisierung besagt, dass Phraseologismen in den Bestand einer Sprache aufgenommen sind.

12 Reproduzierbarkeit meint, dass Phraseologismen nicht jedes Mal neu gebildet, sondern in Form und Bedeutung aus dem Sprachwissen abgerufen werden (vgl. psycholinguistische Festigkeit bei Burger 2007:17 f.).

13 Bei der Koordinierung werden komponentengleiche Phraseologismen zu einem Phraseologismus verbunden.

14 Der Nominalteil eines verbalen Phraseologismus wird abgetrennt und z. B. durch einen Relativsatz wieder aufgenommen.

15 Insofern sich mehrere Phraseologismen auf engem Textraum versammeln, kann es zu Bildbrüchen (Katachresen) kommen, sobald die Bildsphären inkompatibel sind.

16 Ein Phraseologismus kann mit sprachlichen Elementen des Kontextes in Beziehung stehen, z. B. mit Synonymen, Antonymen, Anaphern, Kataphern usw.

17 Verletzt werden die semantischen Selektionsbedingungen eines Phraseologismus z. B., wenn der Kontext nahelegt, zuerst seine wörtliche Bedeutung zu aktualisieren. 
Anführungszeichen, ${ }^{18}$ Phraseologismen als textstrukturierende Prinzipien. ${ }^{19}$ (Da Karlin von phraseologismusrelevanten externen Bezugnahmen kaum Gebrauch macht, gehe ich nur bei entsprechenden Belegen darauf ein.)

Eine Funktionencharakteristik von Phraseologismen muss zwischen pragmatischen Funktionen und Funktionen im künstlerischen Text unterscheiden. Fleischer (1997:218 ff.) versteht unter pragmatischen Funktionen in bestimmten Kommunikationssituationen und bei bestimmten Kommunikationsaufgaben ,die speziellen Wirkungsmöglichkeiten der Phraseologismen zur Unterstützung der Intentionen des Senders“. Insofern können Phraseologismen, oft aufgrund ihres Konnotationspotentials, das soziale Verhältnis zwischen den Kommunikationspartnern indizieren oder die emotional betonte Einstellung des Senders zu dem mitgeteilten Sachverhalt anzeigen und dabei ironisch, scherzhaft, euphemistisch, klischeehaft, bildhaft ${ }^{20}$ etc. auf Empfänger wirken. In künstlerischen Texten, wie dem Untersuchungsroman, sind Phraseologismen „,nur unter dem übergeordneten Gesichtspunkt der ästhetisch-künstlerischen Struktur des Werkes zu beurteilen“" (Fleischer 1997:226). Autoren können die verschiedenen Phraseologismenarten mit ihren spezifischen Eigenschaften ausnutzen, um die ästhetisch-künstlerische Wirkung eines Werkes zu unterstützen. Eine abgeschlossene, stringente Liste von Funktionen für Phraseologismen in künstlerischen Texten existiert nicht - grundlegend zu berücksichtigen sind die Sprachprodukte auf Figurenebene, auf Erzählerebene, ebenso Phraseologismen in hervorgehobenen Textpositionen. Meine nachfolgende formale Analyse durchleuchtet nacheinander die Phraseolexeme sowie die satzwertigen Phraseologismen. In der semantischen und funktionalen Analyse betrachte ich beide Phraseologismusarten gemeinsam. Die Belegdarstellung folgt einem Muster: Beleg, Quelle in WT, Basis, Bedeutung, weitere Erläuterungen z. B. zur Modifikation, literarische Figur als fiktionaler Produzent.

\section{Analyse der Phraseolexeme}

\subsection{Analyse der Phraseolexeme unter formalem Aspekt}

Die Phraseolexeme (nachfolgend PL) lassen sich formal danach ordnen, inwiefern Karlin diese sprachsystemgetreu (in übereinstimmender Form und Bedeutung mit der in Wörterbüchern aufgeführten Basis) oder modifiziert gebraucht.

\subsubsection{Sprachsystemgetreu gebrauchte Phraseolexeme}

Sowohl vollidiomatische PL (vgl. PL 1 bis PL 4, PL 6) als auch teilidiomatische PL (vgl. PL 5, PL 7) gebraucht Karlin sprachsystemgetreu, Person, Numerus, Tempus, Modus und Genus verbi sind dem Kontext angepasst, der wendungsinterne Aktant jmd./jmdm./jmdn. (sofern lexikographiert, habe ich ihn aufgenommen) ist kontextrelevant aktualisiert:

(PL 1) Borgen war ihr zur zweiten Natur geworden. (WT 169), Basis: ${ }^{21} j m d m$. zur zweiten Natur werden $=$,jmdm. selbstverständlich, zur festen Gewohnheit werden` (DUW 2001:1127). [aE über d'Albuquerque]

18 Eine Kommentierung liegt vor, wenn sprachliche oder metasprachliche Mittel eingesetzt sind, um das Verständnis des Phraseologismus zu sichern, z. B. durch Anführungszeichen oder kommunikationssteuernde Lexeme, wie im sprichwörtlichen Sinne, im übertragenen Sinne usw. (ausführlich bei Wotjak 1992:125 ff.).

19 Ein einzelner Phraseologismus kann Textteile oder auch längere Textabschnitte strukturieren, insofern die Texteme semantisch oder formal aus ihm entwickelt werden.

20 Unter „bildhaft“ verstehe ich die visuell-konkreten Vorstellungen, die sprachliche Elemente beim Produzenten oder Rezipienten hervorrufen können. Dagegen meint „bildlich“ die Metaphorizität sprachlicher Elemente.

21 Als „Basis“ bezeichne ich die in Wörterbüchern gespeicherten Ausgangsformen. 
(PL 2) Man dreht den Spieß um und macht den Fluch zum Segen. (WT 94), Basis: den Spieß umdrehen/umkehren $=$ ugs. ${ }^{22}$, mit der gleichen Methode, mit der man angegriffen wird, seinerseits angreifen' (DuR 2011:718). [Ehrental zu Emma]

(PL 3) Andersen und ich, wir sind von der alten Schule (WT 49), Basis: von der alten Schule sein = ,der früher herrschenden Richtung' (DUW 2001:1410). [Rüdiger über sich und Klementines Vater]

(PL 4) Anna hat sich zum Islam bekehren lassen und ist mit einem Mohammedaner durchgegangen. (WT 95), Basis: mit jemandem durchgehen = ugs. ,seinen [Ehe]partner mit einem anderen heimlich verlassen“ (DUW 2001:409). [Klementine zu Primula]

(PL 5) wenns Spitzbuben regnete und der olle Klong ins Haus wollte, stiegen wir ins Boot (WT 48), Basis: es regnet Spitzbuben $=$ analog zu es regnet Schusterjungen/Bindfäden/junge Hunde/Strippen = ugs. ,es regnet sehr stark' (DuR 2011:619). [Rüdiger über sein Leben]

(PL 6) sie ging wie jemand, der die Welt an der Leine hat (WT 78), Basis: jmdn. an der Leine haben/ halten $=$ ugs. ,jmdn. in der Gewalt haben und lenken können'(DuR 2011:483). [Klementine über sich selbst]

(PL 7) War es seine Schuld, daß ihr Frauenherz [...] nach Liebe hungerte (WT 28), Basis: nach Liebe hungern $=$ geh. ,heftiges Verlangen nach Liebe haben' (DUW 2001:808). [Emma über den schottischen Geliebten]

\subsubsection{Modifizierte Phraseolexeme}

Karlin modifiziert die Speicherungen im Phraseolexikon, um ihre literar-ästhetischen Absichten umsetzen zu können. Quantitativ überwiegen die modifizierten, die okkasionellen Phraseolexeme - ein Indiz für die kreative Nutzung des Phraseologiebestandes durch die Romanautorin. Es fällt auf, dass Karlin sowohl mit nur einer Modifikationsart versehene PL einsetzt (einfache Modifikation), aber gleichermaßen PL mit verschiedensten Modifikationsarten gebraucht (mehrfache Modifikation). Zunächst führe ich diejenigen PL auf, die einfach modifiziert sind.

\subsubsection{Interne einfache Modifikation durch semantische Modifikation}

(i) Lexikalische Substitution

Substitution meint den lexikalischen Ersatz einzelner Komponenten des Phraseologismus durch andere, wendungsfremde Lexik. Diese kann auf paradigmatischen semantischen Beziehungen beruhen (synonymische, hyponymische, antonymische, polysemische, homonymische usw. Relationen) oder textgebunden sein, insofern das Substitut lediglich eine semantische Beziehung zum Kontext besitzt (vgl. Wotjak 1992:134; Palm 1995:77 ff.): ${ }^{23}$

(PL 8) daß sie bis zum Hals in Schulden steckt (WT 86), Basis: tief/bis über beide Ohren in Schulden stecken = ugs. ,völlig verschuldet sein' (DUW 2001:1409) = Substitution, paradigmatisch bedingt, Kohyponyme $=$ Hals $>>$ Ohren $^{24}\left[\mathrm{~d}^{\text {‘ Albuquerque über Emma }]}\right.$

(PL 9) Man hatte sich gegenseitig die Zeit verkürzt, hatte sich nichts vorzuwerfen. (WT 28), Basis: $j m d m$. / sich (Dat.) [...] die Zeit vertreiben =,jmdn., sich [mit etw.] für einen bestimmten Zeitraum unterhalten, beschäftigen' (DuR 2011:900) = Substitution paradigmatisch bedingt, partielle Synonyme $=$ verkürzen $>>$ vertreiben. [Emma über den schottischen Geliebten $]$

\footnotetext{
22 Nachfolgend ugs. = umgangssprachliche Stilschicht, geh. = gehobene Stilschicht.

23 Da Modifikationen bei der lexikalischen Substitution Teil des Phraseologismus bleiben, sind sie gleichzeitig fett markiert.

$24 \quad>=$ ersetzt.
} 
(PL 10) Das Leben war interessant, ein Schachspiel, und sie wollte ${ }^{25}$ die geheimen Mächte schachmatt machen und den König heimführen. (WT 30), Basis: jmdn. schachmatt setzen = ugs. ,jmdm. jede Möglichkeit zum Handeln nehmen, ihn als Gegner ausschalten` (DuR 2011:652) = Substitution machen $>>$ setzen, was stilistisch als nicht angemessen zu beurteilen ist. [Emma über Männer im Allgemeinen]

(PL 11) Berge wollte sie verrücken, nicht Kochtöpfe allein! (WT 52), Basis: Berge versetzen (kön$n e n)=$,nahezu Unmögliches vollbringen'. Die Wendung basiert auf der Redensart „der Glaube versetzt Berge“ (NT 1. Kor. 13,2) (DuR 2011:110) = Substitution verrücken $>>$ versetzen. [Bhin über ihre Pläne in Siam]. Bei diesem Beleg verknüpft Karlin zeugmatisch die idiomatisch gebundene und die freie Lesart des Verbs verrücken, so dass eine Katachrese entsteht, die beim Rezipienten wiederum Komik erzeugt. Diese Wirkung resultiert aus dem Konflikt zwischen widersprüchlichen Erfahrungswelten.

(ii) Expansion

Unter Expansion versteht man das Hinzufügen zusätzlicher interner Komponenten zu einem Phraseologismus (vgl. Wotjak 1992:141). In der Fachliteratur werden vor allem adjektivische und Genitivattribute, attributive Präpositionalphrasen sowie die Determinativkomposition ${ }^{26}$ genannt. Hier lässt sich die Liste der Expansionselemente erweitern. Bei der Mehrzahl der expandierten Phraseolexeme fügt Karlin Modaladverbiale hinzu:

(PL 12) Wohl aber schiene es mir angezeigt, Ihrem Koch ein wenig ${ }^{27}$ auf die Finger zu sehen. (WT 84), Basis: jmdm. auf die Finger sehen = ,auf jmdn. [aus Misstrauen] besonders aufpassen“ (DUW 2001: 545) $=$ Expansion durch Modaladverbiale. [Frau Langfar zu d'Albuquerque]

(PL 13) Emma Friedländer hatte die Zähne zusammengebissen in dem Stolz, der selten eine Frau verläßt. (WT 28), Basis: die Zähne zusammenbeißen = ugs. ,ein Höchstmaß an Selbstbeherrschung aufbieten, um etw. sehr Unangenehmes, Schmerzhaftes ertragen zu können' (DUW 2001:1839) = Expansion durch Modaladverbiale. [Emma über sich und den schottischen Geliebten]

(PL 14) Hast du den Rubikon also endlich überschritten? (WT 74), Basis: den Rubikon überschreiten = geh. bildungsspr. ,einen [strategisch] entscheidenden Schritt tun' (nach dem Grenzfluss Rubikon [lat. Rubico] zwischen Italien und Gallia cisalpina, mit dessen Überschreitung Cäsar 49 v. Chr. den Bürgerkrieg begann) (DUW 2001:1327) = Expansion durch Modaladverbiale. [Emma zu Helene über deren Entscheidung für einen Mann]

Die Expansion mit dem Modaladverb mehr und der Vergleichskonjunktion als führt zu einer skalaren Steigerung der phraseologischen Lesart und zum Spiel mit der wörtlichen Lesart:

(PL 15) da rissen Andersen und ich dem Teufel mehr als ein Barthaar aus (WT 48), Basis: dem Teufel ein Barthaar ausreißen ${ }^{28}=$,jmdn. überlisten“ (in keinem Wörterbuch lexikographiert). [Rüdiger über seine Vergangenheit]

(iii) Wechsel von Affirmation - Negation und umgekehrt

Diese Modifikationsart meint die „Negierung von im Phraseologismus usuell nicht negierten Komponenten (Expansion) und umgekehrt (Reduktion)“"(Wotjak 1992:152). Möglich ist auch der Ersatz des usualisierten Negationselements durch Negativoide, wie kaum, schwerlich, selten. Karlin nutzt diese Modifikationsart selten:

25 Die Modalverben betrachte ich als Modifikationen, da sie semantische Abstufungen einbringen.

26 Aus einem Substantiv des Phraseologismus wird mit einem zusätzlichen Lexem ein Determinativkompositum gebildet.

27 Expansionselemente sind mit Unterstreichung gekennzeichnet.

28 Nach dem Märchen der Brüder Grimm ,Der Teufel mit den drei goldenen Haaren`, die Barthaare werden dem Teufel von dessen Großmutter ausgerissen, damit die drei Rätsel für das Glückskind gelöst werden können, das Glückskind nicht getötet, sondern mit der Königstochter vermählt wird. 
(PL 16) Sie hatte von den Besucherinnen keine Notiz genommen (WT 68), Basis: Notiz von jmdm., etw. nehmen = jmdm., einer Sache Beachtung, Aufmerksamkeit schenken' (DuR 2011:555). [Anna beim Besuch von Klementine und Primula]

(iv) Semantische Modifikationen, die einen Assoziationshof eröffnen

Beim Beleg (PL 17) mit der Seele unter dem Arm trägt ein Assoziationshof, aufgefasst als semantische Modifikation, zur Semantisierung von Phraseologismen bei:

(PL 17) da rissen Andersen und ich dem Teufel mehr als ein Barthaar aus, und wenns Spitzbuben regnete und der olle Klong ins Haus wollte, stiegen wir ins Boot und ruderten hinab, wo die übrigen Deutschen mit der Seele unter dem Arm saßen, und veranstalteten eine Stimmbänderverrenkung (WT 48). [Vater Rüdiger über seine Vergangenheit]

Im gleichen Satz gebraucht der Deutsche Rüdiger zwei isotopiekonstituierende PL. Der Phraseologismus mit der Seele unter dem Arm besitzt keinen Eintrag im DuR 2011. Es handelt sich möglicherweise um eine Modifikation des Phraseologismus den Kopf unterm Arm tragen (ugs.) = ,sehr krank sein' (DuR 2011:436). Kopf steht im genannten PL für die kranke Physis des Menschen - Seele könnte hier analog für die kranke, von der Materie gelöste Psyche der Siedler in den Kolonien stehen. Vom Lexem Seele aus eröffnet sich semantisch-inferenziell ein Assoziationshof, der das syntagmainterne Lexem Teufel und weitere Phraseologismen einbezieht: auf etw. erpicht sein wie der Teufel auf die arme Seele; hinter etw. her sein wie der Teufel hinter der armen Seele = ugs. ,auf etw. versessen sein` (DuR 2011:767). Die Phraseologismenhäufung macht das Sprachporträt von Rüdiger auffällig, sie charakterisiert ihn an dieser Romanstelle als humorvolle Figur. Aufgrund der Verwandtschaft der Bilder (mit Teufelsfigur) kann man von einer „Anziehung“ der Phraseologismen sprechen. ${ }^{29}$

\subsubsection{Interne einfache Modifikation durch strukturelle Modifikation}

Eine interne „einfache“ Modifikation durch strukturelle Modifikation findet sich bei Karlin lediglich als Reduktion ${ }^{30}$ und als Nominalisierung: ${ }^{31}$

(PL 18) „Mit dir könnte man den Boden aufwischen!“ (WT 37). [Emma zu Helene]. Im DuR 2011 findet sich kein Eintrag. Als optionale Basis setze ich: mit jmdm. wie mit einem Waschlappen den Boden aufwischen. Im Kontext verwendet Emma kohäsiv das okkasionelle Adjektiv waschlappig zur Benennung einer abwertenden Eigenschaft Helenes - Waschlappen aktualisiert hier die Lesart ugs.-abwertend ,ein Feigling, ein Schwächling‘ (DUW 2001:1779), d. h., Emma sieht Helene als zu schwach an, ihre Männerprobleme selbstbewusst lösen zu können. $=$ Reduktion wie mit einem Waschlappen.

(PL 19) bis sie das Warum einer Handlung ans Licht gebracht (WT 26), Basis: etw. ans Licht bringen/ziehen/zerren/holen = ,etw. [Verheimlichtes] an die Öffentlichkeit bringen' (DUW 2001:1017) = Reduktion hat. [aE über Emma]. Den geh. Gebrauch des infiniten Partizips II anstelle einer finiten Verbform habe ich (vgl. Pohl 2019a:159) bezüglich des Romans als individualstilistische Besonderheit Karlins gekennzeichnet (vgl. ebenso PL 23).

Reduktionen können bei Karlin so weit gehen, dass nur ein Sinnträger des PL genannt wird:

(PL 20) wenn man in Backfischüberschwenglichkeit von, zum Fressen gern' gesprochen hätte (WT 20), Basis: jmdn. zum Fressen gern haben = ugs. scherzh. ,sehr gern haben“ (DUW 2001:575)

\footnotetext{
29 Nach Richter-Vapaatalo (2007:104) kann eine „Anziehung“ von Phraseologismen bei Häufungen durch die Synonymität von Phraseologismen, aber auch ,,durch die Verwandtschaft des Bildes oder der Struktur oder auch lediglich durch ihren phraseologischen Charakter“ zustandekommen.

30 Mit Wotjak verstehe ich unter Reduktion „kontextbedingte Weglassungen im wendungsinternen Komponentenbestand, nicht aber Reduktionen im (wendungsexternen) Aktantenpotential von verbalen PL“(Wotjak 1992:146; Auszeichnungen originalgetreu, $\mathrm{PL}=$ Phraseolexem).

31 Die Nominalisierung erfasst häufig die im Phraseologismus enthaltenen Verben.
} 
$=$ Reduktion jmdn. ... haben. [Prinzessin Bhin über ihren Namen, der übersetzt ,Granatapfel` bedeutet]

(PL 21) und ihre bezüglichen Väter es oft für angezeigt hielten, eine, Wucht' zu verabreichen (WT 46), Basis: eine Wucht (Tracht Prügel) kriegen = landschaftlich salopp ,heftige Schläge, Prügel bekommen' (DUW 2001:1831) = Reduktion kriegen/bekommen, gleichzeitig liegt ein Patiens-AgensTausch vor. [Rüdiger über seinen eigenen und Klementines Vater]

(PL 22) ein Sitzen zwischen zwei Stühlen (WT 17), Basis: sich zwischen zwei Stühle setzen = , sich zwei Möglichkeiten o. Ä. gleichermaßen verscherzen“ (DUW 2001:1540) = Modifikation durch Wortbildung: Nominalisierung sich setzen >> ein Sitzen. [Herbert zu Klementine über Mischrassen]

\subsubsection{Interne mehrfache Modifikation}

In der Mehrzahl der Belege setzt Karlin mehrfache Modifikationen ein, bevorzugt die Expansion in Kombination mit verschiedenen anderen Modifikationsarten:

(PL 23) Auch eine arme Eurasierin, die zwischen zwei Stühlen auf dem Boden sitzengeblieben. (WT 138), Basis: sich zwischen zwei Stühle setzen = , sich zwei Möglichkeiten o.Ä. gleichermaßen verscherzen' (DUW 2001:1540) = Expansion durch Lokaladverbiale auf dem Boden + sitzenbleiben (perfektiv) $\gg>$ sich setzen (imperfektiv) $=$ Aktionsartwechsel ${ }^{32}+$ Reduktion ist $($ Reduktion und geh. Stilschichtzugehörigkeit wie bei (PL 19)). [Klementine über d'Albuquerque]

(PL 24) weil sie immer eine braune Frau haben können, mit der man keine Geschichten zu machen braucht (WT 6), Basis: keine langen Geschichten machen = ,keine Umstände machen' (DUW 2001:639) = Expansion braucht + Reduktion langen. [Emma über weiße Männer in Siam]

(PL 25) Der Schotte war eine Perle im schmerzhaften Rosenkranz der Erfahrungen, den sie, als Weib, herabbeten mußte. (WT 29), Basis: einen Rosenkranz beten = ,Reihung von Gebeten (bes. Vaterunser und Ave Maria), die in bestimmter Abfolge gebetet werden' (DUW 2001:1323) $=3 \times$ Expansion (adj. Attribut + Genitivattribut + Modalverb musste) + Abtrennung mit pronominaler Wiederaufnahme, der unbestimmte Artikel einen entfällt wegen der Aufnahme des adjektivischen Attributs schmerzhaft + Wortbildung Präfigierung mit pejorativer Wertung bei herabbeten. [Emma über den schottischen Geliebten]

(PL 26) Wie man sein Bestes verteidigt, wie man sich aufbewahrt wie einen Sonntagshut, und sich dann plötzlich einmal, ohne Leidenschaft, um eine Pferdefeige wegwirft ... Immer an den Unrechten natürlich, wie Perlen vor die Schweine. (WT 29), Basis: Perlen vor die Säue werfen = ugs. ,etwas Wertvolles jmdm. anbieten, geben, der es nicht zu schätzen, zu würdigen weiß (verbreitet durch Matthäus 7,6; DuR 2011:577) = Expansion mehrerer Adverbiale // dann plötzlich einmal, // ohne Leidenschaft, // um eine Pferdefeige // ... Immer an den Unrechten natürlich // + Substitution Schweine $>$ Säue + das unecht reflexive Verb werfen ist im grammatischen Status modifiziert, prädiktabel ist der Anschluss des Vergleichs an das reflexiv gebrauchte Verb sich wegwerfen. [Emma über ihr bisheriges Leben]

(PL 27) Ihre dunkle Begleiterin [...] drückte selbst Klementine in erhöhtem Maße den Stempel des Halbbluts auf (WT 44), Basis: jmdm., einer Sache seinen Stempel aufdrücken = ,jmdn., etw. auf charakteristische Weise beeinflussen' (DuR 2011:733) = Expansionen: Partikel selbst + Modaladverbiale in erhöhtem Maße + Genitivattribut des Halbbluts. [Klementine über Anna Rüdiger]

(PL 28) Selbst die Missionare, die auf die Wogen des Rassenhasses das Öl der Bruderliebe gossen, vermieden ängstlich Mischehen. (WT 47), Basis: Öl auf die Wogen gießen =, vermittelnd, ausgleichend, besänftigend [in eine Auseinandersetzung] eingreifen' (Wellen werden durch daraufgegossenes Öl geglättet) (DUW 2001:1161) = Expansion durch 2x Genitivattribut des Rassenhasses, der

32 Vgl. zu Aktionsarten okkasioneller Verben in WT bei Pohl (2019b). 
Bruderliebe + grammatische Modifikation (best. Artikel $>>$ Nullartikel). [aE über Mischehen] (PL 29) Die junge Prinzessin wandte sich stumm ab und opferte dem Meergott zu Lande. (WT 53), Basis: Neptun opfern (Neptun ist der römische Gott des Meeres) = ugs. scherzhaft ,(wegen Seekrankheit) sich über die Reling gebeugt übergeben' (DuR 2011:549) = Substitution, synonymisch Meergott $\gg$ Neptun + grammatische Modifikation (Nullartikel $\gg$ best. Artikel/obligatorisch) + Expansion durch präpositionales Attribut $z u$ Lande. [aE über Prinzessin Bhin, nachdem sie Ekelerregendes in der siamesischen Küche (zu Lande) sah]

(PL 30) wir saßen alle knietief im Sumpf (WT 60), Basis: im Sumpf sitzen = , sich in einer fast ausweglosen Lage befinden' (nach DuR 2011:752) = Expansion Modaladverbiale knietief + Expansion Indefinitpronomen alle (betont Ganzheitlichkeit des Subjekts wir, keiner ist ausgeschlossen). [Ehrental über seine Handelsgeschäfte]

(PL 31) hatte noch genug Mutteraugen übrig (WT 15), Basis: Augen im Kopf haben = ugs. ,etw. durchschauen, beurteilen können“ (DuR 2011:73) = Expansion durch Modaladverbien noch genug + Expansion durch DK Mutter- + lexikalische Substitution übrig haben , als Rest noch vorhanden; verbleibend; restlich“ >> haben ,besitzen' (DUW 2001:1632) + Reduktion im Kopf. [Frau Langfar über sich, wie sie Primula schützt]

(PL 32) Wir Männer stehen gern mit dem Rücken gegen das Feuer. (WT 13), Basis: mit dem Rücken an der/zur Wand stehen/kämpfen = , in einer äußerst schwierigen Situation, in einer Lage, in der man sich energisch wehren, verteidigen muss' (DUW 2001:1328) = textgebundene Substitution gegen das Feuer $>>$ an der/zur Wand + Expansion Modaladverbiale gern. [Herr Langfar humorvoll über sich]

Andere Modifikationsarten (im Unterschied zur Expansion) kombiniert Karlin seltener:

(PL 33) Wie albern, aus dem Regen unter die Traufe zu treten! (WT 107), Basis: aus dem/vom Regen in die Traufe kommen = ugs. , aus einer unangenehmen, schwierigen Lage in eine noch schlimmere geraten'(DUW 2001:1289) = grammatische Modifikation: Präpositionenwechsel in $>>$ unter + lexikalische Substitution (treten $>>$ kommen, gelangen/geraten'). [Emma über ihre Liaison mit Herbert]

\subsubsection{Von Karlin kreierte ungewöhnliche Phraseolexeme}

Im Roman finden sich drei ungewöhnliche verbale PL, die lexikographisch nicht belegt sind:

(PL 34) Keine Sklavin, geschweige denn eine Prinzessin, schießt so aus den Gelenken. (WT 56) = ,sich gehen lassen', ,einen heftigen Zornesausbruch bekommen'. Eine siamesische Geliebte von Bhins Vater, des siamesischen Prinzen, ereifert sich über den Zornesausbruch der siamesischen Prinzessin Bhin, nachdem diese das Konkubinentum ihres Vaters durchschaut hat. Möglicherweise gibt Karlin die Übersetzung eines siamesischen Phraseologismus wieder. Semantisch gestützt wird das ungewöhnliche Phraseolexem mittels der direkten Rede weiterer Geliebter: „Nicht einmal eine verpeitschte und bevorzugte Verstoßene oder eine zurückgesetzte, unbefriedigte Hauptfrau, die ihrem Gatten viele Söhne geboren hat und hohe Ansprüche stellen darf, läßt sich je so gehen!" (WT 56; Sperrdruck originalgetreu).

(PL 35) Sie fuhr von Ecke zu Winkel, zupfte an Kissen und Deckchen. (WT 41) =, sich schnell und aufgeregt im Zimmer hin und her bewegen, von einer Ecke zur anderen'. [aE über die aufgeregte Helene, nachdem sie einen Heiratsantrag erhalten hat]. Möglicherweise benutzt Karlin den elliptischen Ausdruck von einer Ecke zur anderen rennen in der Bedeutung, aufgeregt hin und herrennen ' als Basis. Wenig ausdrucksstark, redundant wäre die Vollform von einer Ecke zur anderen Ecke, so dass Karlin für Ecke ein partielles Synonym einsetzt: von Ecke zu Winkel. Unter Ecke versteht man ,in einem Winkel aneinanderstoßende Flächen', unter Winkel, eine Ecke von innen gesehen' (vgl. DUW 2001:418). Vom Verb fahren ist im PL ein Semem aktualisiert $=$, sich rasch, hastig in eine bestimmte Richtung, an eine bestimmte Stelle bewegen“ (DUW 2001:513). 
(PL 36) der Futterwüterich plant eine Pumpe (WT 9) =, vorhaben, Geld zu pumpen/borgen'. [Emma über d'Albuquerque]. Hier liegt eine humorvolle Modifikation des teilidiomatischen PL Geld pumpen vor. Die Lexeme Pumpe und pumpen (Semem 2) sind semantisch in diesem Kontext nicht aufeinander beziehbar. Pumpe besitzt die Sememe (1) ,Vorrichtung, Gerät zum An- oder Absaugen von Flüssigkeiten oder Gasen', des Weiteren (2),(salopp) Herz' und (3) ,Spritze, mit der Rauschgift injiziert wird', pumpen bedeutet (1) ,mit einer Pumpe etwas befördern' und (salopp) (2) ,bei, von jmdm. etwas borgen, leihen' (vgl. DUW 2001:1255). Vorhandene Substantive zu pumpen (Semem 2) sind Pump und Pumpen. Der Humor ergibt sich aus der Gleichzeitigkeit zweier unverträglicher Erfahrungswelten und ruft bei Rezipienten Schmunzeln oder Lächeln hervor.

Fazit zur formalen Analyse der Phraseolexeme: Bei den untersuchten 36 Phraseolexemen nutzt Karlin vorrangig das Muster der verbalen PL, auch die neugebildeten PL besitzen ein Verb als Kernlexem. Über Gründe dieser Wahl kann nur spekuliert werden, offensichtlich lassen sie sich gut in einen Satz einbauen und kreativ modifizieren. Von den 36 PL insgesamt werden 26 PL modifiziert, was rein quantitativ auffällig ist:

\begin{tabular}{|c|c|c|c|c|}
\hline & Modifikationsart & Gesamtzahl & $\begin{array}{l}\text { davon idiomatisierte } \\
\text { PL }\end{array}$ & $\begin{array}{l}\text { davon nichtidio- } \\
\text { matisierte PL }\end{array}$ \\
\hline \multirow[t]{4}{*}{$\begin{array}{l}\text { einfache semantische } \\
\text { Modifikation }\end{array}$} & $\begin{array}{l}\text { lexikalische } \\
\text { Substitution }\end{array}$ & 4 & $\begin{array}{l}2 \\
\text { (PL 10,11) }\end{array}$ & $\begin{array}{l}2 \\
(\mathrm{PL} 8,9)\end{array}$ \\
\hline & Expansion & 4 & $\begin{array}{l}4 \\
\text { (PL 12-15) }\end{array}$ & - \\
\hline & $\begin{array}{l}\text { Wechsel Affirmation } \\
\text { - Negation }\end{array}$ & 1 & $\begin{array}{l}1 \\
\text { (PL 16) }\end{array}$ & - \\
\hline & $\begin{array}{l}\text { mit Assoziationshof } \\
\text { (Häufung) }\end{array}$ & 1 & $\begin{array}{l}1 \\
\text { (PL 17) }\end{array}$ & - \\
\hline $\begin{array}{l}\text { einfache strukturelle } \\
\text { Modifikation }\end{array}$ & Reduktion & 5 & $\begin{array}{l}4 \\
(\mathrm{PL} \mathrm{18}, 19,21,22)\end{array}$ & $\begin{array}{l}1 \\
\text { (PL 20) }\end{array}$ \\
\hline $\begin{array}{l}\text { mehrfache Modifi- } \\
\text { kation (semantisch + } \\
\text { strukturell) }\end{array}$ & & 11 & $\begin{array}{l}10 \\
\text { (PL 23, 24, 26-33) }\end{array}$ & $\begin{array}{l}1 \\
\text { (PL 25) }\end{array}$ \\
\hline Gesamtanzahl & & 26 & 22 & 4 \\
\hline
\end{tabular}

Tab. 1: Modifikationsarten der Phraseolexeme

Aus der tabellarischen Auflistung der modifizierten PL lassen sich mit gebotener Vorsicht folgende Erkenntnisse ableiten:

(i) Die einfache semantische sowie die mehrfache Modifikation der PL sind die bevorzugten Modifikationsarten Karlins, um die PL in diesem Roman einzubetten.

(ii) Bei der mehrfachen Modifikation bevorzugt Karlin die Expansion in Kombination mit verschiedenen Modifikationsarten.

(iii) Dass Karlin vorrangig idiomatisierte PL modifiziert (22 von insgesamt 26), zeigt, dass sie den dadurch erreichten semantischen Mehrwert (vgl. Kap. 5) zur Beziehungsgestaltung mit dem Adressaten einsetzt. Die idiomatisierten PL werden an den Kontext „,angepasst“, womit zudem Verständnissicherung beabsichtigt ist.

Eine formale textstrukturierende Funktion der Phraseologismen, wie bei Wolff/Ehrhardt (2017) für Karlin herausgearbeitet, z. B. Kapiteleinleitung, lässt sich für diesen Roman nicht belegen. Dennoch verfügen die Phraseologismen über eine semantisch-strukturierende Funktion hinsichtlich von Textbedeutung und Textbotschaft (vgl. Kap. 5.3). 


\section{Analyse der satzwertigen Phraseologismen}

Nachfolgend werden Sprichwörter und Geflügelte Worte, zusammengefasst zu satzwertigen Phraseologismen, untersucht. Man versteht unter Sprichwörtern verallgemeinernde abgeschlossene Sätze, die mit unbekanntem Verfasser überliefert sind und Lebenserfahrungen, -weisheiten mit meist lehrhafter Tendenz vermitteln. Unter funktionalem Aspekt handelt es sich um, ,allgemeine Aussagen oder Urteile, mit denen eine gegebene Situation erklärt, eingeordnet, beurteilt wird“ (Handbuch 1982:39).$^{33}$ Durch die Zitation eines Geflügelten Wortes mit bekanntem Autor wird eine vergleichbare Redesituation ,in einen kulturhistorischen Kontext“" gesetzt (Palm 1995:5). Voraussetzung für ein positives Leseerlebnis ist die Kenntnis der Bildungsmodalitäten. Diese allgemeinen Einschätzungen können durch konkrete Analysen des literarischen Textes spezifiziert werden.

\subsection{Analyse der satzwertigen Phraseologismen unter formalem Aspekt}

Die von mir ermittelten 37 satzwertigen Phraseologismen setzen sich zusammen aus 29 Sprichwörtern (mit und ohne Nachweis in Sprichwörterlexika) und 8 Geflügelten Worten. Beide Subgruppen untersuche ich zunächst gesondert.

Die Sprichwörter (nachfolgend SW) lassen sich formal nach dem Kriterium untergliedern, inwiefern sie von Karlin sprachsystemgetreu, d. h. in übereinstimmender Form und Bedeutung mit der Speicherung im Sprachsystem (Nachweis in Sprichwörterlexika), gebraucht werden.

\subsubsection{Sprachsystemgetreu gebrauchte Sprichwörter}

(SW 1) Hier liegt der Hase im Pfeffer. (WT 134), Basis: Da/hier liegt der Hase im Pfeffer. (ugs.) (DuR 2011:338). ${ }^{34}$ [Ehrental über seine Reisen]

(SW 2) Sage mir, mit wem du umgehst ... (WT 90), Basis: Sage mir, mit wem du umgehst, und ich sage dir, wer du bist. (SpriL 1989:481). [Fräulein d'Albuquerque über Helene]

Die Bekanntheit des Sprichwortes setzt Karlin voraus, das Fragment sollte beim Rezipienten die Inferenz auf den vollständigen Wortlaut zulassen.

(SW 3) wissend, daß man die Menschen nehmen mußte, wie sie waren, und nicht, wie sie sein könnten oder sein sollten (WT 91), Basis: Man muss die Menschen nehmen, wie sie sind. (SpriL 1989:397). [Frau Langfar]

(SW 4) Nur gute Saat konnte gute Ernte bringen. (WT 147), Basis: Gute Saat, gute Ernte. // Wie die Saat, so die Ernte. (SpriL 1989:478). [Emma über ihr Leben]

(SW 5) Wo ein Wille ist, ist ein Weg. (WT 94), Basis: Wo ein Wille ist, da ist auch ein Weg. (SpriL 1989:674). [Dr. Nathfield zu Primula]

Die Belege (SW 4) und (SW 5) unterscheiden sich formal und semantisch marginal im Vergleich zur Basis, dennoch ordne ich die Belege hier ein. Bei (SW 4) ist die konditionale Beziehung der Basis verbalisiert, bei (SW 5) hebt in der Basis zusätzlich die Partikel auch das Bedingte hervor, das Lokaladverb da im Hauptsatz verweist anaphorisch auf den Nebensatz.

33 Von den echten Sprichwörtern mit kulturhistorischem Hintergrund sind Gemeinplätze semantisch unterschieden: Ein Gemeinplatz formuliert oft nicht bildhaft „eine Tatsache, die aufgrund allgemeinen Weltwissens selbstverständlich ist“ (Burger 2007:42). Burger fasst Sprichwörter und Gemeinplätze zur Gruppe der Topischen Formeln zusammen, als Subgruppe der satzwertigen Phraseologismen (vgl. Burger 2007:41). In meiner Untersuchung führe ich die Gemeinplätze mit bei den Sprichwörtern auf, da es strukturelle und funktionale Übereinstimmungen gibt, z. B. bei der Bewertung oder als Rechtfertigung von Handlungen und Situationen (vgl. Burger 2007:42).

34 Vgl. die Etymologie: „Das sprachliche Bild bezieht sich auf den zubereiteten Hasenbraten in einer scharf gewürzten Soße, dem sogenannten ,Hasenpfeffer', und veranschaulicht damit den vermutlich ursprünglichen Sinn der Wendung, nämlich dass jmd. aus einer bestimmten Lage nicht mehr herauskommt“ (DuR 2011:338). 


\subsubsection{Sprichwörter mit fragmentarischen semantisch-formalen Entsprechungen im Phraseo- lexikon}

Die in dieser Gruppe von Karlin kreierten Sprichwörter lassen sich semantisch-formal auf ein lexikographisch erfasstes Sprichwort beziehen, sie werden jedoch nicht in ihrer ursprünglichen Form verwendet, sondern modifiziert. Anhand von formal gleichen oder variierten „Ankerwörtern“, die es sowohl in der Basis als auch in der Kreation (hier fett markiert) gibt, kann ein Rezipient ein usualisiertes Sprichwort inferieren. Lexikalische Veränderungen lasse ich unberücksichtigt. Mitunter sind die syntaktische Struktur der Basis sowie das abstrakte Strukturmodell übernommen (vgl. [SW 10]: Wer X tut, der tut/erleidet $\mathrm{Y}$ ).

(SW 6) Mit welchem Recht setzte das Schicksal einem Grenzen, zu enge Grenzen? (WT 93), Basis: Seinem Schicksal kann niemand entgehen. (SpriL 1989:498). [Primula über sich selbst]

(SW 7) Ja, Liebe - das Kraut der Unsterblichkeit. (WT 178), Basis: Gegen die Liebe ist kein Kraut gewachsen. (SpriL 1989:367). [aE über Dr. Nathfield und Primula]

(SW 8) Die Frucht ist lockend, aber sitzt nicht ausgerechnet in den schönsten Früchten der dickste Wurm? (WT 19), Basis: Im schönsten Apfel sitzt der Wurm. (SpriL 1989:688). [Herbert über Siam]

(SW 9) Alles auf Erden war nur Werden oder Vergehen. (WT 164), Basis: Was bald wird, vergeht auch bald. (SpriL 1989:670). [Klementines Großmutter über Klementines Tod]

(SW 10) Wer mit dem Feuer spielt, muß auf den Blasen sitzen. (WT 73), Basis: Wer die Hand ins Feuer steckt, verbrennt sich die Finger. (SpriL 1989:158). [Emma zur Freundin Helene]

(SW 11) Erst das Untersuchen der Wunde, dann erst den Balsam des Vergessens darauf. Was nicht ausgebrannt wird, heilt nicht. (WT 27), Basis: Die Wunden, die man heilen will, muss man erst aufdecken. (SpriL 1989:686). [Emma über ihr Verhältnis zum schottischen Geliebten]

(SW 12) Er hatte recht gehandelt und war doch schuldig geworden. (WT 172), Basis: Wer recht handelt, braucht das Licht nicht zu scheuen. / Recht tun ist keine Sünde. (SpriL 1989:460). [Herbert über die Tatsache, dass er Klementine abgewiesen hat]

(SW 13) Alles geht vorüber! (WT 113), Basis: Es ist alles vergänglich. (SpriL 1989:30). [Klementines Großmutter zu Klementine, nachdem deren Mutter sie geschlagen hat]

(SW 14) Er war die Welt auf- und abgerutscht wie der sprichwörtliche Stein, der kein Moos sammelt. (WT 46), Basis: Auf dem rollenden Stein wächst kein Moos. (SpriL 1989:402). [Rüdiger über sich selbst]

(SW 15) Vater sprach ja auch immer davon, daß man das eigene Leid am schnellsten überwand, wenn man fremdes Leid milderte. (WT 123), Basis: Trösten ist leichter als leiden. (SpriL 1989:357).

[Primula über sich selbst]

Werden die relativ stabilen Sprichwörter so stark wie bei Karlin modifiziert, ist dies besonders auffällig. Den phraseologischen Charakter der Sprichwörter betonen z. B. metasprachliche Kommentare in (SW 14) sprichwörtlich und in (SW 8) ausgerechnet, womit auf eine bekannte zugrundeliegende Basis des Sprichworts verwiesen wird. Das verallgemeinernde Präsens dominiert, lediglich bei (SW 12) ist das Plusquamperfekt und bei (SW 15) das Präteritum genutzt, um eine „Passung“ an den Kontext zu erreichen. Beide verbalisierte Handlungen liegen vor der Sprechzeit der literarischen Figuren.

\subsubsection{Von Karlin kreierte Sprichwörter ohne Entsprechungen im Phraseolexikon}

(SW 16) Ich muß mich irgendwo verankern, denn ein treibendes Schiff wirft der Sturm gegen die Klippen. (WT 122). [Dr. Nathfield über sich] 
(SW 17) Sie wollte nicht an sich denken - wer an sich selbst dachte, lief ewig wie ein Esel in einer Tretmühle rund und rund und sah nur sich selbst auf die Füße. (WT 118). [Emma über sich und Herbert]

(SW 18) Selbst die längste Straße hat eine Biegung, und die deine wird kommen. (WT 118). [Emma zur Freundin Helene]

(SW 19) Im Liebesspiel sehen die direkten Teilnehmer immer die Tragik, die Zuschauer das Lustspiel oder die Farce. (WT 118). [Emma über Helenes Verhalten Männern gegenüber]

(SW 20) In den Verkehr von Mann und Frau fließt immer etwas ein, was weniger oder mehr als Freundschaft ist. (WT 26). [Emma über den schottischen Geliebten]

(SW 21) Alles ist dort schön, wo es klar hingehört. (WT 19). [Kühlbrück über Siam]

(SW 22) Jeder Esel kann eine Tretmühle laufen machen, doch nur ein Löwe, ein Tiger setzt mutig über eine hohe Mauer hinweg und holt sich, was er begehrt. (WT 94). [Dr. Nathfield zu Primula]

(SW 23) Kein Halm rührt sich, wenn ein Frosch durch den Dschungel springt, aber wenn ein Tiger brüllt, zittern Sträucher, Vögel, Tiere. (WT 101). [Siamesischer Prinz über seinen hohen Status]

(SW 24) Viel wird von dem begehrt, dem viel gegeben wurde. (WT 101). [Siamesischer Prinz über seinen hohen Status und seine Verpflichtungen]

(SW 25) Wird nicht manche Frucht um soviel süßer, nachdem der erste Frost gefallen? (WT 176). [Dr. Nathfield über Primula und ihre Lepraerkrankung]

(SW 26) So war das Leben: die alten Blätter mußten vom Baum fallen, um den neuen Schößlingen Raum zu machen. (WT 176). [Ehepaar Langfar über das Leben]

(SW 27) Furchtlos wie seit einem Vierteljahrhundert, gingen sie der kommenden Nacht entgegen, denn war das Dunkel nicht ein Meer von Sternen und war die Nacht nicht nur das Tor des Morgens? (WT 177). [Ehepaar Langfar über das Leben]

\subsubsection{Nutzung von Strukturmustern}

Bei wenigen Sprichwörtern nutzt Karlin bekannte abstrakte Strukturmuster der deutschen Sprache, füllt diese jedoch mit okkasioneller Lexik auf: phraseologisches Muster: ohne - kein/e: $:^{35}$

(SW 28) Ohne Wärme, ohne Licht kam nichts zur Reife. (WT 91) = Ohne Wärme/Licht keine Reife. [Primula über sich selbst]

phraseologisches Muster: je - desto: ${ }^{36}$

(SW 29) Je mehr ein Mann und ein Hund nach einem Lieblingsbissen springen müssen, desto ausdauernder werden sie ... (WT 42) // Je mehr wir geben, desto stärker lieben wir ihn oder das, dem wir geben. (WT 27). [Emma über die Männer]

Fazit zu den Sprichwörtern: Listet man die Sprichwörter nach dem formalen Kriterium auf, ergibt sich das folgende Bild: Fünf Sprichwörter sind sprachsystemgetreu eingesetzt, zehn Sprichwörter sind modifiziert, zwölf Sprichwörter sind von Karlin ohne Wörterbuchentsprechung kreiert, zwei Sprichwortmuster sind mit okkasioneller Lexik gefüllt. Allein diese Auflistung verweist darauf, dass Karlin sprachkreativ mit der deutschen Sprache umgeht, denn von den 29 aufgefundenen Sprich-

35 Das Modell ohne - kein/e (ohne X kein Y) dient der Angabe eines Konditionalverhältnisses: Wenn/Falls keine Wärme da ist, dann kommt auch keine Reife.

36 Das Modell je - desto (je mehr X desto mehr Y) dient der Angabe einer Proportion. 
wörtern werden lediglich fünf in ihrer Wörterbuchentsprechung ohne Modifikationen eingesetzt. Bei (SW 2) zitiert Karlin nur den Anfang, sie rechnet mit der Sprichwortkenntnis ihrer Leser.

Bei zehn Sprichwörtern setzt Karlin Modifikationen gegenüber einem Basissprichwort ein, wobei sie sich häufig mehrfacher Modifikationen bedient. Die Modifizierungen erbringen generell für das literarische Werk einen semantischen Mehrwert, den ich hier für die dominierenden Modifikationsarten beschreibe:

Dass Karlin mehrheitlich Expansionen einsetzt, ist der Autorintention geschuldet,

- indem sie gegenüber der Basis mehr Informationen übermitteln möchte, wie bei (SW 15): Vater sprach ja auch immer davon, daß man das eigene Leid am schnellsten überwand, wenn man fremdes Leid milderte. (WT 123);

- indem sie eigene Erfahrungswerte einbringen möchte, wie bei (SW 8): Die Frucht ist lockend, aber sitzt nicht ausgerechnet in den schönsten Früchten der dickste Wurm? (WT 19);

- indem sie das Sprichwort textual an eine literarische Figur bindet, wie bei (SW 14): Er war die Welt auf- und abgerutscht wie der sprichwörtliche Stein, der kein Moos sammelt. (WT 46). Rüdiger sieht sich als tüchtigen, umtriebigen Menschen, jedoch wird er im Roman als Weißer charakterisiert, der ,veröstelt" ist und sein Leben im europäischen Verständnis nicht im Griff hat;

- indem sie die All-Regionalität als Aspekt der Allgemeingültigkeit des Sprichworts betonen möchte, wie bei (SW 9): Alles auf Erden war nur Werden oder Vergehen. (WT 164).

Bei den Belegen mit Reduktion sticht das Beispiel (SW 12) hervor. Hier wird an das Fragment des Sprichworts eine stilistische Antithese angeschlossen, die aufgrund der Reduktion des Sprichworts intensiv wirkt: Er hatte recht gehandelt und war doch schuldig geworden. (WT 172).

Von den Sprichwörtern mit Substitution hebt sich (SW 10) mit umfänglicher Substitution ab. Lediglich das Stützlexem Feuer ist nicht substituiert, das okkasionelle Sprichwort erscheint aufgrund des Szenenwechsels drastisch und bildhafter als die Basis: Wer mit dem Feuer spielt, muß auf den Blasen sitzen. (WT 73).

Die Sprichwörter (SW 6) bis (SW 27) sowie (SW 28) und (SW 29) (letztere mit indizierenden Konstruktionselementen lexikographierter Sprichwörter) bezeichne ich als okkasionelle Sprichwörter, die Karlin nach den Merkmalen von Sprichwörtern kreiert hat: Es handelt sich um verallgemeinernde, meist polysituative satzwertige Mikrotexte mit einem abgeschlossenen Gedanken, um eine kurze Spruchform, die bildhaft und mit lehrhaftem Anspruch Karlins Lebenserfahrungen vermittelt. Dass nicht in jedem Fall das verallgemeinernde Präsens verwendet wird, ist der Anbindung an den Text geschuldet. Die Verallgemeinerungen, wodurch Sprichwörter im Sprachbewusstsein kognitiv verankert sind, kommen zum Ausdruck,

- indem Karlin den unbestimmten Artikel verwendet, z. B. (SW 16) Ich muß mich irgendwo verankern, denn ein treibendes Schiff wirft der Sturm gegen die Klippen.

(WT 122), oder auch dessen Negierung als Indefinitpronomen kein, z. B. (SW 23) Kein Halm rührt sich, wenn ein Frosch durch den Dschungel springt, aber wenn ein Tiger brüllt, zittern Sträucher, Vögel, Tiere. (WT 101);

- indem Karlin sehr bewusst Indefinitpronomen nutzt, wie jeder in (SW 22), alles in (SW 21), manche in (SW 25) oder viel (in SW 24);

- indem Karlin Adjektive mit unbestimmtem Zeitbezug verwendet, wie ewig in (SW 17);

- indem Karlin das Temporaladverb immer verwendet, z. B. (SW 19) und (SW 20) In den Verkehr von Mann und Frau fließt immer etwas ein, was weniger oder mehr als Freundschaft ist. (WT 26).

Die Mehrzahl der okkasionellen Sprichwörter ist der literarischen Figur Emma in den Mund gelegt, was den Befund stützt, dass Karlin diese literarische Figur mit beeindruckender Lebenserfahrung 
und Sprachkreativität ausstattet (vgl. Pohl 2019a) (vgl. unten Funktionen der Phraseologismen im Sprachporträt von Emma).

Bei den Geflügelten Worten (nachfolgend GW) ist für Karlin typisch, dass sie weder Quelle noch Autornamen angibt (vgl. ähnlichen Befund bei Wolff/Ehrhardt 2017). Ob sie die Rezipienten bewusst im Unklaren lässt oder ob sie von Rezipienten mit hoher Sprachkompetenz ausgeht, kann nicht entschieden werden. Die aufgespürten Quellen offenbaren Karlins favorisierte Interessengebiete. So zitiert sie in vier Geflügelten Worten aus der Bibel:

(GW 30) Grundsätze gelten im Großen wie im Kleinen (WT 90), Basis: Wer im Kleinen (nicht) treu ist, der ist auch im Großen (nicht) treu. (Nach Lukas 16,10) (SpriL 1989:315). [Frau Langfar über das Leben]

(GW 31) Dein Volk soll mein Volk und dein Gott soll mein Gott sein (WT 175; WT 176). Primulas Trauspruch, von Langfar bei der Trauung formuliert: ,, - denn wohin du gehst, dorthin will auch ich gehen; und wo du wohnst, da will auch ich wohnen; dein Volk soll mein Volk und dein Gott soll mein Gott sein ... "/ (Ruth 1,16; nach der Lutherbibel). [Herr Langfar]

(GW 32) In unseres Vaters Haus gibt es viele Wohnungen (WT 91) Nach Johannes 14,2: „Im Haus meines Vaters gibt es viele Wohnungen. Wenn es nicht so wäre, hätte ich euch dann gesagt: Ich gehe, um einen Platz für euch vorzubereiten?“ (vgl. Bibel in der Einheitsübersetzung). [Herr Langfar]

(GW 33) Alles braucht seine Zeit, besonders im Osten. (WT 84), Basis: Alles hat seine Zeit. (Nach Prediger Salomo 3,1) // Alles zu seiner Zeit. (Nach Prediger Salomo 3,11) (SpriL 1989:30). [Frau Langfar über das siamesische Rechtswesen]

Hierzu gehört der Spruch des aus der Kirchenliteratur bekannten Kirchenvaters Hieronymus $(347-420)$ :

(GW 34) Irren ist menschlich! (WT 90) (Hieronymus, Epistolae) (SpriL 1989:284). [Frau Langfar zu Fräulein d'Albuquerque]

Karlin bezieht des Weiteren Zitate aus der Klassischen und aus der Musikliteratur mit ein:

(GW 35) Raum ist in der kleinsten Hütte (WT 131) Zitat aus Friedrich Schillers (1776-1805) Gedicht ,Der Jüngling am Bache‘ (DuR 2011:610). [Ehrental spottet über Herbert]

(GW 36) Liebe ist eine Himmelsmacht (WT 174) Aus der Operette ,Der Zigeunerbaron“ von Johann Strauss (Sohn), Uraufführung 1885 in Wien. [aE über Herbert und Emma]

Und schließlich zitiert Karlin einen Staatsmann, den Gründervater der Vereinigten Staaten:

(GW 37) Ach was, waschlappig! Dreimal gut ist ... (WT 38), Basis: Dreimal umziehen (umgezogen) ist so gut wie einmal abgebrannt. (Nach Benjamin Franklin (1706-1790), Way to wealth' (SpriL 1989:111). [Emma über die Freundin Helene]. Hier wird lediglich das Basis-Satzmuster genutzt mit dem Ankerwort Dreimal. Das GW wird situativ auf Helenes Gutmütigkeit bezogen - ein ,schlimmer Ausgang" wird ihr von Emma prophezeit und soll so vom Rezipienten inferiert werden.

Aufgrund der verwendeten Geflügelten Worte kann man insgesamt annehmen, dass sich Karlin in den Herkunftsquellen, insbesondere in den überwiegend religiösen Texten, auskannte und dass sie sich zeitgemäß-aktuell informiert hat.

Unter formalem Aspekt ist auch nach der architektonisch-kompositorischen Einbettung der satzwertigen Phraseologismen zu fragen. Im Roman treten die Phraseologismen als Teil der Rede des auktorialen Erzählers oder als Teil erlebter und direkter Rede literarischer Figuren auf. Architektonisch besitzen sie keine hervorgehobene Position, kompositorisch tragen sie über die semantischen Vernetzungen zur Betonung der Autorintention bei (vgl. unten). 


\section{Analyse der Phraseolexeme und satzwertigen Phraseologismen unter se- mantischem Aspekt}

Unter semantischem Aspekt ist zu ermitteln, welchen semantischen Mehrwert die Phraseologismen einbringen. Dieser ist $u$. a. dadurch zu erfassen, indem zugrundeliegende Konzepte (hier am Beispiel der satzwertigen Phraseologismen) ermittelt werden, die über Denotatives hinausgehenden Konnotationen betrachtet werden, semantische Vernetzungen aufgesucht werden und indem die Metaphorizität bildlicher Phraseologismen hinterfragt wird.

\subsection{Zugrundeliegende Konzepte der satzwertigen Phraseologismen}

Bezüglich zugrundeliegender Konzepte orientiere ich mich an Hessky/Ettinger (1997): „Unter der ideographischen Beschreibung des Lexikons verstehe ich seine Repräsentation in Richtung ,Konzept $>$ Zeichen', den Ausgangspunkt der Lexikon-Darstellung bilden also nicht die Welt, sondern [...] die Ideen der Menschen über die Welt" (Hessky / Ettinger 1997:XXI; Schreibung originalgetreu). Interessant ist die Auswahl der Konzepte, ${ }^{37}$ die den Äußerungen einzelner literarischer Figuren unterlegt sind, und die Frage, ob sie zur „Sicht auf die Welt“ der jeweiligen literarischen Figuren ${ }^{38}$ „passen“. Exemplarisch wähle ich einige literarische Figuren aus.

Die satzwertigen Phraseologismen in Emmas Sprachgebrauch sind thematisch dreigeteilt: zum einen Äußerungen gegenüber der Freundin Helene, zum anderen ihrem eigenen Verhalten Männern gegenüber und schließlich über ihr eigenes Leben. Helene gegenüber ist ihre emotional-wertende Stellungnahme ambivalent. Einerseits liegt das Konzept GERINGSCHÄTzUnG (GW 37) ${ }^{39}$ zugrunde, andererseits ERmutigung (SW 18), aber auch NeCKerei (SW 10) und Gute LaUne (SW 19) ihr gegenüber. Emmas Haltung gegenüber Männern im Allgemeinen ist basiert durch die Konzepte GERINGSCHÄTZUNG (SW 29), BEHERRSCHEN einer Situation (SW 11) sowie realen EINSCHÄTZENS (SW 20), aber auch von PfLichtbewUSSTSEIN (SW 17) und schließlich, ihr gesamtes Leben betreffend, von einem Sinn FÜR REAlitäTEn (SW 4). Die ermittelten Konzepte sind mit Emmas Charakterisierung (vgl. Pohl 2019a) kompatibel, die Sprichwörter stimmig ausgewählt.

Die Konzepte, die sich aus den Belegen zum Missionarsehepaar Langfar eruieren lassen, ergeben ein geschlossenes Bild ihrer Eigenschaften, wie GelassenHeIt (GW 33), (SW 3), (SW 26), (SW 27), Beurteilen (GW 33) und Standhaftigkeit / ZuversichtLichKeIt (GW 30), (GW 31), (GW 32).

Auch zum Weißen Dr. Nathfield, der um Primula wirbt und sie heiratet, hat Karlin Konzepte gewählt, die seine Eigenschaften und seine Einstellung zu Mitmenschen ausdrücken, wie OPTIMISMUS (SW 5), ENTSChlossenheit / TATKRAFt (SW 16), (SW 22) und Zuneigung / Liebe (SW 25).

Der Deutsche Herbert, der Mischehen als „Verbrechen“ (WT 172) bezeichnet (vgl. Pohl 2019a, b), bringt in den von ihm verwendeten satzwertigen Phraseologismen zu Beginn der Romanhandlung Misstrauen / BezWeifeln (SW 8) zum Ausdruck, bis er am Romanende, nachdem die von ihm abgewiesene Mischlingsfrau Klementine verstorben ist, BEZWEIFELN mit der Einstellung SCHULD (SW 12) mischt.

Den vom aE geäußerten satzwertigen Phraseologismen (GW 36) und (SW 7) sind die Konzepte ANERKENNUNG / ACHTUNG unterlegt, die sich entsprechend der Autorintention auf reinrassige Liebesverhältnisse beziehen (Herbert und Emma, Dr. Nathfield und Primula).

Im Handeln von Klementines siamesischer Großmutter spielen die Konzepte BeJaHEN, SchlaUes, KLUGes Handeln und Vorsicht (SW 13), (SW 9) eine Rolle.

Zusammenfassend lässt sich aus den satzwertigen Phraseologismen schließen, dass Karlin eine überschaubare Zahl an Konzepten vermittelt (vgl. Konzeptesammlung von Hessky/Ettinger 1997: V-IX). Die Konzepte der im Roman eingesetzten satzwertigen Phraseologismen gehören zu

\footnotetext{
37 Namen für Konzepte entnehme ich in Großschreibung den ideographischen Bezeichnungen eines anthropozentrischen Weltbildes im Verständnis von Hessky/Ettinger (vgl. 1997:XXXV).

38 Vgl. die Charakteristik der literarischen Figuren in Pohl (2019a).

39 In der Klammer nach dem Konzept gebe ich den entsprechenden Beleg an.
} 
folgenden Großgruppen: C Eigenschaften des Menschen, D Einstellung, Beziehung zu den Mitmenschen, zur Umwelt, E Menschliches Handeln. Nicht in satzwertigen Phraseologismen sind von Karlin ausgedrückt die Großgruppen: A Aussehen des Menschen, B Zustand des Menschen, F Einschätzung einer Lage, eines Sachverhalts, G Umwelt - Außenwelt und H Situationsgebundene Phraseologismen. Karlin betont folglich mittels der Konzepte Eigenschaften der literarischen Figuren, ihre Beziehungen zueinander und zur Umwelt sowie ihr Handeln im Rahmen von Paarbeziehungen im interkulturellen Raum Siam, was mit der Autorintention des Romans korreliert (vgl. Pohl 2019a, b, c). Affin sind diesbezüglich besonders die Konzepte, welche die Autorin den Äußerungen der literarischen Figuren Emma, Herbert und des aE unterlegt.

\subsection{Semantische Zusatzinformationen mittels Konnotationen}

Unter Konnotationen verstehe ich semantische Informationen, die über eine denotative Bedeutung hinausgehen, mit einem Formativ verbunden sind und kommunikative Rahmenbedingungen der Sprachverwendung signalisieren. Hier betrachte ich die emotionale Höhenlage der Phraseologismen, die durch Stilschicht- und Stilfärbungskonnotationen semantischen Mehrwert einbringt. Der größte Teil der im Roman eingesetzten Phraseologismen ist stilschichtlich ,ugs. "/ugs.-salopp“ konnotiert, wodurch konventionell Nähe zur Alltagsrede erreicht wird, wie z. B.: und wenns Spitzbuben regnete (PL5), gestützt durch die Kontamination von wenn und es. Jedoch nutzt Karlin auch mehrmals die geh. Stilschicht, wie in (PL 19) bis sie das Warum einer Handlung ans Licht gebracht. Zu beobachten ist, dass die geh. Stilschicht der Phraseologismen häufig erst durch einen Transfer des Kontextes entsteht, z. B. mittels des Konjunktivs II und des veralteten Verbgebrauchs schiene angezeigt im Hauptsatz: Wohl aber schiene es mir angezeigt, Ihrem Koch ein wenig auf die Finger zu sehen. (PL 12). Auch eine grundlegende romanrelevante Aussage des aE ist stilschichtlich geh. konnotiert: Selbst die Missionare, die auf die Wogen des Rassenhasses das Öl der Bruderliebe gossen, vermieden ängstlich Mischehen. (PL 28). Die geh. Stilschicht scheint Karlin die adäquate Einbettung ihres vermeintlich ,hohen Romanthemas“, sie möchte dieses aufgrund ihres interiorisierten Wertesystems als wertvoll, als anspruchsvoll verstanden wissen (vgl. Pohl 2019b:265).

Gleichermaßen setzt Karlin Stilfärbungen ein. Der aE kann durchaus ,humorvoll` sein, wie bei der Periphrase des Sich-Übergebens von Prinzessin Bhin: Die junge Prinzessin wandte sich stumm $a b$ und opferte dem Meergott zu Lande. (PL 29). Stilfärbungen setzt die Autorin weiterhin dazu ein, einzelne literarische Figuren zu charakterisieren. Eine Konnotation ,bildungssprachlich` besitzt Emmas Frage an ihre Freundin Helene: Hast du den Rubikon also endlich überschritten? (PL 14). Die Geflügelten Worte im Roman lassen sich nur zum Teil eindeutig als ,bildungssprachlich` konnotiert bewerten, wie die Rede des Missionars Langfar: In unseres Vaters Haus gibt es viele Wohnungen (GW 32). Andere Geflügelte Worte sind nach meinem Dafürhalten in den allgemein gebräuchlichen Wortschatz übergegangen, so dass die Konnotation ,bildungssprachlich' neutralisiert ist, wie in der direkten Rede der Missionarsgattin: Irren ist menschlich! (GW 34). Eine Stilfärbung ,ironisch lässt sich für die indirekte Rede des Deutschen Rüdiger über die Erziehungsmethoden seines und Andersens Vater annehmen, da ihre bezüglichen Väter es oft für angezeigt hielten, eine ,Wucht' zu verabreichen (PL 21). Die ironische Brechung kommt aufgrund der semantischen Inkompatibilität des geh. Verbs verabreichen und des ugs. Nomens Wucht (,Schläge, Dresche`) zustande (vgl. zur ironischen Brechung Palm 1995:4).

Zusammengefasst ergibt sich, dass Karlin Konnotationen vor allem durch die stilschichtlichen Zuordnungen ,ugs.-salopp“ und ,geh.' einbringt, gesenkte Stilschichten gebraucht sie nicht. Stilfärbungen charakterisieren das Sprachporträt des aE als ,humorvoll ' und ,scherzhaft', das Sprachporträt des Deutschen Rüdiger mit ,ironischer Brechung' und die Sprachporträts Emmas und des Missionars Langfar teilweise als ,bildungssprachlich“. Letztere Zuordnungen sind kein „Spiel mit Bildung“" (vgl. Palm 1995:5), sondern dienen unter romanrelevant-ästhetischen Aspekten der Sprachporträtierung: Beim Missionar Langfar indiziert ,bildungssprachlich' eine höhere Schulbildung. An der zentralen literarischen Figur Emma möchte Karlin verdeutlichen, wie aus dem einstigen Klein- 
bauernmädchen eine Dame (WT 37) wird, zu der nach Karlins Auffassung ein gehobensprachlicher Duktus mit der Stilfärbung, bildungssprachlich‘ gehört (vgl. Pohl 2019a:154 ff.).

\subsection{Semantischer Mehrwert mittels semantischer Vernetzungen}

Semantische Vernetzungen von Phraseologismen lassen sich insbesondere zu zwei themenrelevanten Semantikfeldern feststellen: zum ,Bild von der weißen vs. von der braunen Frau' und zu ,Rasse und Mischehe'. Aufgrund der isotopischen Vernetzung relevanter Phraseologismen entstehen Ketten, welche die Textbedeutung und die Textbotschaft strukturieren sowie die Wertigkeit der phraseologischen Referenzen erhöhen.

\subsubsection{Phraseologisches Semantikfeld ,Bild von der weißen vs. von der braunen Frau“}

Diesbezüglich sind die Reden von Emma, der siamesischen Prinzessin Bhin, Klementine und vom $\mathrm{aE}$ vernetzt. Quantitativ überwiegt die Rede der Figur Emma. Braune Frauen sind ihrer Meinung nach solche, mit denen man keine Geschichten zu machen braucht (PL 24), sie böten sich unterwürfig, damit verurteilenswert, den weißen Männern in Siam an. Das Bild der weißen Frau, das Karlin offensichtlich in der ehrgeizigen, gesellschaftliche Konventionen übertretenden, damit menschlich erscheinenden Emma verkörpert sieht, ist mithilfe der PL in seiner widersprüchlichen Komplexität erfahrbar: Ihr Frauenherz hungerte nach Liebe (PL 7), aber sie hatte bei all ihren schlechten Erfahrungen mit Männern die Zähne zusammengebissen in dem Stolz, der selten eine Frau verläßt (PL 13). Männer im Allgemeinen sind in Emmas Vorstellung eine Perle im schmerzhaften Rosenkranz der Erfahrungen, den sie, als Weib, herabbeten mußte (PL 25). Über ihr bisheriges Leben resümiert sie: Wie man sein Bestes verteidigt, wie man sich aufbewahrt wie einen Sonntagshut, und sich dann plötzlich einmal, ohne Leidenschaft, um eine Pferdefeige wegwirft ... Immer an den Unrechten natürlich, wie Perlen vor die Schweine (PL 26). Nun wollte sie nicht aus dem Regen unter die Traufe (...) treten (PL 33), sondern den König heimführen (PL 10), den reichsten und angesehensten Mann, und die Geliebte auf Zeit des Weißen Ehrental werden. In der positiven Bewertung durch den aE widerspiegelt sich Emmas wesentliche Charaktereigenschaft: Emma gibt sich mit keiner Situation zufrieden, sie forscht nach, bis sie das Warum einer Handlung ans Licht gebracht (PL 19).

Eine Semantisierung von ,Ehrgeiz ' und ,Entscheidungsfreudigkeit' ist nachzuvollziehen im Sprachporträt der Halbsiamesin Klementine: sie ging wie jemand, der die Welt an der Leine hat (PL 6) und der siamesischen Prinzessin Bhin: Berge wollte sie verrücken, nicht Kochtöpfe allein! (PL 11). Diese Phraseologismen widersprechen allerdings Karlins Intention, Mischlings- bzw. braune Frauen nicht zu positiv zu bewerten (vgl. Stamm 2012).

\subsubsection{Phraseologisches Semantikfeld ,Rasse und Mischehe'}

Karlins Roman erscheint 1933, intendiert von Karlins ethisch-moralischer Auffassung von Rassenreinheit vs. Rassenmischung. Karlins mehrfach belegte rassische Ansicht, dass jede gemischtrassige Beziehung im Unglück endet (vgl. Šlibar 2006; Stamm 2012; Pohl 2019a), wird semantisch in einigen vernetzten Phraseologismen eingefangen. Das Semantikfeld wird genährt mittels Phraseologismen in den Äußerungen der literarischen Figuren Klementine, des Deutschen Herbert und des aE. Als dominante Aussage erscheint die Feststellung des aE über Mischehen mit dem Beleg eines (vermeintlichen) Autoritätsbeweises: Selbst die Missionare, die auf die Wogen des Rassenhasses das Öl der Bruderliebe gossen, vermieden ängstlich Mischehen. (PL 28). Das Hohelied der Liebe „singt" der aE über reinrassige Beziehungen, wie über Dr. Nathfield und Primula: Ja, Liebe - das Kraut der Unsterblichkeit. (SW 7) und über Herbert und Emma: Liebe ist eine Himmelsmacht. (GW 36).

Ein nicht reinrassiger Mischling zu sein, wird von den literarischen Figuren als gesellschaftlicher Makel gesehen: Ihre dunkle Begleiterin [...] drückte selbst Klementine in erhöhtem Maße 
den Stempel des Halbbluts auf (PL 27). Ein Mischling, verscherze sich zwei Möglichkeiten o.Ä. gleichermaßen', weil er keiner Rasse eindeutig angehöre: Es sei nach Herberts Warnung zu Romanbeginn ein Sitzen zwischen zwei Stühlen (PL 22). Die metaphorische Aussage erscheint Karlin so wesentlich, das sie diese von der Mischlingsfrau Klementine über Fräulein d'Albuquerque variiert wiederholen lässt: Auch eine arme Eurasierin, die zwischen zwei Stühlen auf dem Boden sitzengeblieben. (PL 23).

Der Deutsche Herbert formuliert die semantisch stärkste Abwertung von Mischheiraten. Nachdem er die Mischlingsfrau Klementine abgewiesen hat, stirbt sie an Cholera und an ,gebrochenem Herzen": Mischheiraten waren so unheilvoll, waren ein Verbrechen an der Rasse. Er hatte recht gehandelt und war doch schuldig geworden ... (SW 12). Die literarische Figur Herbert ist überzeugt davon, dass sie im Sinne der Reinrassigkeit richtig gehandelt hätte - zum Adjektiv recht lässt sich das Substantiv Recht inferieren mit der Bedeutung, Gesamtheit der staatlich festgelegten bzw. anerkannten Normen des menschlichen bzw. gesellschaftlichen Verhaltens' (DUW 2001:1282). Inwiefern sich Herbert schuldig fühlt, kann mit dem Blick auf Klementines Tod lediglich vermutet werden.

\subsection{Zur Metaphorizität bildlicher Phraseologismen}

Bei der Vielzahl der bildlichen Phraseologismen scheint mir sinnvoll, sich auf die von Karlin neugebildeten 14 Sprichwörter (SW 16 bis SW 29) zu konzentrieren, bei denen die Autorin verschiedenartigste semantische Motivationen nutzt. In (SW 21) ist ein Gemeinplatz formuliert, der keinen metaphorisch übertragbaren Bildspender besitzt: Alles ist dort schön, wo es klar hingehört. (WT 19). Im Roman jedoch besitzt der Gemeinplatz einen konkreten Referenten: Die Figur Kühlbrück wertet damit das multikulturelle Siam ab. (SW 24) steht einem Gemeinplatz nahe. Bei den restlichen zwölf Sprichwörtern kann man überschaubare Bildspendbereiche ausmachen, die Karlin aus dem Vorstellungsbereich ihrer potentiellen Leser ausgewählt hat, um das Leseerlebnis positiv zu beeinflussen (vgl. einen ähnlichen Befund bei Wolff/Ehrhardt 2017:225). Für (SW 22) und (SW 23) mit den Lexemen Löwe, Tiger und Dschungel könnte man annehmen, dass sich Karlin an siamesischasiatischen Sprichwort-Vorbildern oder an der siamesisch-asiatischen Lebenswelt orientiert hat, vgl.: (SW 23) Kein Halm rührt sich, wenn ein Frosch durch den Dschungel springt, aber wenn ein Tiger brüllt, zittern Sträucher, Vögel, Tiere. (WT 101). Als Bildspendbereich dient hauptsächlich die Natur i. w. S. wie bei den folgenden Sprichwörtern auch (SW 17), (SW 25), (SW 26) und (SW 28) Ohne Wärme, ohne Licht kam nichts zur Reife. (WT 91). Einen quantitativ etwas geringeren Bildspendbereich Freundschaft/Liebe besitzen (SW 19), (SW 20), auch (SW 29) Je mehr ein Mann und ein Hund nach einem Lieblingsbissen springen müssen, desto ausdauernder werden sie ... (WT 42). Das Verhalten von Tieren wählt Karlin als Bildspendbereich für (SW 17), (SW 29). Und schließlich spendet die Seefahrt das Bild in (SW 16), der Tag-Nacht-Rhythmus das Bild für (SW 27) Furchtlos wie seit einem Vierteljahrhundert, gingen sie [Missionarsehepaar Langfar - I. P.] der kommenden Nacht entgegen, denn war das Dunkel nicht ein Meer von Sternen und war die Nacht nicht nur das Tor des Morgens? (WT 177). Die Bildspendbereiche in absteigender Reihenfolge verdeutlichen, dass Karlin usuelle Bildspendbereiche ausgewählt und keine außergewöhnlichen metaphorischen Phraseologismen kreiert hat.

\section{Analyse der Phraseolexeme und satzwertigen Phraseologismen unter funktionalem Aspekt}

Funktionale Aspekte im Verständnis einer relationalen Stilistik zu berücksichtigen, bedeutet, dass Phraseologismen in der Determination von Kommunikationsbereichen und Textsorten beschrieben werden. Sinnvoll erscheint beim literarischen Text, das stilistische phraseologische Potential hinsichtlich seiner Funktionen bei der Sachverhaltsdarstellung, der Beziehungsgestaltung und der Selbstdarstellung zu ermitteln. 
(i) Funktionen der Phraseologismen bei der Sachverhaltsdarstellung

Karlin vermag einen Teil der Phraseologismen sinnkonstituierend so einzusetzen, dass diese textkohärent funktionieren. Aufgrund ihres Vernetzungspotentials z. B. entstehen die semantischen Felder zu ,Bild von der weißen vs. von der braunen Frau' und ,Rasse und Mischehe'. Beide semantische Isotopieebenen sind Tragpfeiler sowohl für die Textbedeutung als auch für die Textbotschaft (vgl. Kap. 1).

Im Zusammenhang damit steht eine argumentierende Funktion, indem Karlin den aE unter Berufung auf „Romanautoritäten“ (die Missionare) die Hauptthese formulieren lässt: Selbst die Missionare, die auf die Wogen des Rassenhasses das Öl der Bruderliebe gossen, vermieden ängstlich Mischehen. (PL 28). Das für den Roman wesentliche Textem zur Vermittlung des moralischphilosophischen Anliegens wird phraseologisch herausgehoben.

Konkretisierende Funktionen haben Phraseologismen im Roman, indem Situationen charakterisiert werden, z. B. wird die Eigenschaft Frau Langfars als Mutter mittels Modifikation eingebracht: (PL 31) hatte noch genug Mutteraugen übrig (WT 15). Die konkretisierende Funktion ist bei Karlin häufig mit einer intensivierenden Funktion gekoppelt, indem mehrfach modifizierte Phraseologismen eingesetzt sind, z. B. (PL 27) Ihre dunkle Begleiterin [...] drückte selbst Klementine in erhöhtem Maße den Stempel des Halbbluts auf (WT 44).

Die Zeichnung von Sprachporträts der literarischen Figuren kann als charakterisierende Phraseologismenfunktion eingeordnet werden. Die folgende Tabelle listet auf, welche literarischen Figuren welche Phraseologismenarten in welcher Anzahl verwenden:

\begin{tabular}{|l|l|l|l|}
\hline $\begin{array}{l}\text { literarische Figuren } \\
\text { (Figurenebene/Erzählerebene) }\end{array}$ & $\begin{array}{l}\text { Anzahl der gebrauch- } \\
\text { ten Phraseolexeme }\end{array}$ & $\begin{array}{l}\text { Anzahl der gebrauchten satz- } \\
\text { wertigen Phraseologismen }\end{array}$ & $\begin{array}{l}\text { Gesamt- } \\
\text { anzahl }\end{array}$ \\
\hline Emma Friedländer & 11 & 9 (davon 1 GW) & 20 \\
\hline auktorialer Erzähler & 6 & 2 (davon 1 GW) & 8 \\
\hline $\begin{array}{l}\text { Rüdiger (Vater der Halbsiame- } \\
\text { sin Anna) }\end{array}$ & 5 & 1 & 6 \\
\hline Missionarsehepaar Langfar & 3 & 8 (davon 5 GW) & $11(: 2)^{40}$ \\
\hline Dr. Norbert Nathfield & & 4 & 4 \\
\hline Moses Ehrental & 2 & 2 (davon 1 GW) & 4 \\
\hline Primula Langfar & & 3 & 3 \\
\hline $\begin{array}{l}\text { Herbert Lange (deutscher } \\
\text { Unternehmer) }\end{array}$ & 1 & 2 & 3 \\
\hline Klementine & 3 & 2 & 3 \\
\hline Klementines Großmutter & & 2 & 2 \\
\hline Vater der siam. Prinzessin Bhin & & 1 & 2 \\
\hline Fräulein d'Albuquerque & 1 & & 2 \\
\hline Prinzessin Bhin & 2 & 1 & 2 \\
\hline $\begin{array}{l}\text { Kühlbrück (deutscher Unter- } \\
\text { nehmer) }\end{array}$ & & gesamt: 37 & 1 \\
\hline Anna Rüdiger & 1 & & 1 \\
\hline $\begin{array}{l}\text { siamesische Geliebte von } \\
\text { Klementines Vater }\end{array}$ & 1 & gesamt: 36 & gesamt: 73 \\
\hline
\end{tabular}

Tab. 2: Quantitative Auflistung des Phraseologismengebrauchs einzelner literarischer Figuren

40 Das Ehepaar Langfar führe ich zusammen auf, da es funktional hinsichtlich des Phraseologismengebrauchs keine Unterschiede zwischen den Ehepartnern gibt. 
Die im Zusammenhang mit dem phraseologischen Semantikfeld ,Rasse und Mischehe“ beschriebenen sieben Phraseologismen stellen ca. 10 \% der Phraseolexeme und satzwertigen Phraseologismen dar - ihnen kommt hinsichtlich der Autorintention eine herausragende Rolle zu.

Bezüglich der Figurencharakterisierung (den aE zähle ich hier dazu) sind verschiedene Aspekte zu unterscheiden. Die Phraseologismen helfen mit, das Sprachporträt der literarischen Figuren zu zeichnen. Aus der quantitativen Zuordnung (vgl. Tabelle 2) könnte man vorsichtig schließen, dass relativ viele Figuren des Romans (ausgenommen ist Emmas Freundin Helene) sowie der aE Phraseologismen benutzen, was auf eine diesbezüglich geringe Differenzierung ihrer Sprachporträts durch die Autorin hindeutet. Man kann jedoch unterscheiden, dass äußerst aktive, mitunter humorvolle Figuren des Romans, wie Emma, Vater Rüdiger, das Ehepaar Langfar und der aE, mehr Phraseologismen verwenden als die unauffälligeren Romanfiguren. Generell indizieren die ugs.-saloppen Phraseologismen als Elemente des Sprachporträts eine gewisse Nähe zur mündlichen Alltagssprache, was insbesondere durch die modifizierten Sprichwörter, aber auch durch die Sprichwortfragmente, so bei d'Albuquerque (SW 2) und Emma (GW 37), geleistet wird. Der Gebrauch ugs. Phraseologismen verleiht den Figuren den Charakter sprachangemessenen Verhaltens in mündlichen Kommunikationssituationen. Die charakterisierende Funktion steht im Zusammenhang mit der stilistischen Funktion der Intensivierung, die sich z. B. in der Phraseologismenhäufung beim Deutschen Rüdiger (vgl. PL 17 und öfter) zeigt. Darüber hinaus nutzt Karlin konnotative Bedeutungselemente, um Figuren und den aE hinsichtlich emotionaler Höhenlagen ihrer Äußerungen zu charakterisieren (vgl. oben Punkt 5.2). Hervorzuheben ist insgesamt, dass Karlin die PL und die satzwertigen Phraseologismen in hoher Kompatibilität zum Charakter der literarischen Figuren ausgewählt und eingesetzt hat.

Stärker erscheint ein zweiter Aspekt bezüglich der Figurencharakterisierung: So erfüllen die eingesetzten Sprichwörter eine ideographische Funktion, indem die literarischen Figuren Einschätzungen von sich bzw. Einstellungen zu sich selbst (vgl. Emma, Rüdiger, Primula), emotional bedingte Verhaltensweisen zu anderen Menschen (vgl. Emma, Ehrental, Herbert, Dr. Nathfield, Missionarsehepaar Langfar, siamesischer Prinz) oder emotional bedingte eigene Handlungen (vgl. Fräulein d'Albuquerque, Klementines Großmutter, Herbert, Dr. Nathfield) zum Ausdruck bringen. Auf diese Weise erfüllen die Sprichwörter inklusive der unterlegten Konzepte zwar ebenfalls eine sprachporträtierende Funktion, gleichzeitig kommen in den Sprichwörtern jedoch romanrelevante Einstellungen zum Ausdruck.

(ii) Funktionen der Phraseologismen bei der Gestaltung der Beziehung zum Adressaten

Die Funktion der Beziehungsgestaltung besteht generell darin, Adressaten für das literarische Thema aufzuschließen. Dies geschieht im Roman auf vielfältige Weise. Auch wenn der aE nicht identisch mit Karlin ist, hat er mit Bezug auf die Textbotschaft und gegenüber dem Adressaten eine pointiert autoritätsvertretende Funktion. Die wesentliche Textbotschaft, die Bewertung von Mischehen, ist ihm in den Mund gelegt, er zeigt sich dem Leser gegenüber als Autorität, vgl. (PL 28). Andererseits tritt der aE humorvoll, scherzhaft auf, er möchte offensichtlich Vertraulichkeit stiften, was gleichermaßen durch die ugs. konnotierten Phraseologismen bewirkt wird. Eine Vertrautheit mit dem Adressaten wird simuliert, die gleichzeitig Lockerheit im Umgang der Figuren untereinander, aber auch ihm gegenüber assoziiert, z. B. (PL 14) und (PL 29).

Die von Karlin eingesetzten Phraseologismen besitzen ohne Zweifel veranschaulichende Funktion, was für Rezipienten aufgrund der Phantasieanregung Unterhaltungswert impliziert, z. B. (PL 32) Wir Männer stehen gern mit dem Rücken gegen das Feuer. (WT 13).

Insbesondere diejenigen Phraseologismen, welche die isotopiestiftenden Felder (,Bild von der weißen vs. von der braunen Frau' und ,Rasse und Mischehe') konstituieren, haben Einfluss auf die Emotionen der Adressaten. Die Frage, ob Rezipienten aufgrund der Emotionalisierungsfunktion von Phraseologismen der Textbotschaft folgen oder diese ablehnen, muss offen bleiben.

Karlin geht vermutlich von einer Phraseologiekompetenz ihrer Leser aus, denn sie verwendet überwiegend bekannte, nicht stark idiomatisierte Phraseologismen und sie kommentiert nur in we- 
nigen Belegen metasprachlich, vgl. (SW 14) Er war die Welt auf-und abgerutscht wie der sprichwörtliche Stein, der kein Moos sammelt. (WT 46).

(iii) Funktionen der Phraseologismen bei der Gestaltung der Selbstdarstellung der Autorin Beim aE ist die Selbstdarstellung der Autorin, bezogen auf die Textbotschaft, am stärksten relevant, indem die Ablehnung von Mischehen zum Ausdruck kommt (vgl. PL 28). Diese Bewertung hat in Bezug auf den Rezipienten eine Emotionalisierungsfunktion, Karlin möchte die Rezipienten gefühlsmäßig für sich gewinnen.

Die Einstellung Karlins zur Sprache zeigt sich insbesondere im kreativen Sprachgebrauch der Phraseologismen:

(i) Karlin wählt nur solche Phraseologismen aus, die der Sachverhaltsdarstellung und der Beziehungsgestaltung angemessen sind (ugs., geh.), sie verwendet keine Phraseologismen aus einer gesenkten Stilschicht. Dies bedeutet, dass sie einen ihrer Ansicht nach ,gehobenen“ Romaninhalt in einer ugs. bis geh. Stilschicht autorintendiert verbalisieren möchte.

(ii) Karlin bildet neue, ungewöhnliche PL und satzwertige Phraseologismen. Die Neubildungen der satzwertigen Phraseologismen mögen nicht in jedem Fall den Status von Sprichwörtern besitzen, eher von individuellen Lebensweisheiten, jedoch greift Karlin bewusst auf Wesensmerkmale von Sprichwörtern zurück.

(iii) Insbesondere in Modifikationen von PL und satzwertigen Phraseologismen zeigt sich Karlins sprachliche Kreativität. Einerseits ermöglichen Modifikationen Einbettungen in den Kontext, andererseits sind sie das Merkmal Karlinscher Sprachbewusstheit und Sprachbeherrschung.

\section{7. $\quad$ Fazit}

Bei den von Karlin gebrauchten Phraseologismen dominieren die phraseologischen Vergleichskonstruktionen, die einer eigenen Untersuchung vorbehalten bleiben müssen. Die von mir untersuchten PL und satzwertigen Phraseologismen, die an zweiter Stelle des Gebrauchs stehen, machen ca. 27 \% der Gesamtanzahl der im Roman eingesetzten Phraseologismen aus.

(1) In der Analyse formaler Aspekte wird deutlich, dass von den 36 PL 80 \% okkasionell von Karlin gebildet sind (inklusive neugebildeter PL), von den 37 satzwertigen Phraseologismen entsprechen $65 \%$ okkasionellen Bildungen (inklusive neugebildeter satzwertiger Phraseologismen). Die Belege beweisen, dass Karlin beim Phraseologismengebrauch vorrangig die Muster okkasioneller Bildungen nutzt, wobei sie Okkasionalität mittels semantischer einfacher sowie mehrfacher Modifikation der Basen (vorrangig über die Expansion), mittels des Ausnutzens von Ankerwörtern, mittels der Bildung ungewöhnlicher PL und satzwertiger Phraseologismen erreicht. Autorin-spezifisch scheint zu sein, dass Karlin Lebensweisheiten mithilfe der Wesensmerkmale von Sprichwörtern verbalisiert.

(2) Die Analyse semantischer Aspekte erlaubt einige Schlussfolgerungen. So sind die den Äußerungen literarischer Figuren zugrundeliegenden Konzepte klug ausgewählt, sie sind ,passgetreu“ zum Handeln der literarischen Figuren im Rahmen der Paarbeziehungen im interkulturellen Raum Siam. Ergänzend und stimmig sind Phraseologismen mit den Konnotationen ,ugs.' sowie ,humorvoll'/,scherzhaft', ,ironisch gebrochen“ und ,bildungssprachlich“ zur Sprachporträtierung genutzt. Die Semantikfelder ,Bild von der weißen vs. von der braunen Frau' und ,Rasse und Mischehe' konstituieren kohärente Isotopieebenen und heben Karlins Referenz auf Textbedeutung und Textbotschaft hervor. Bezüglich der Metaphorizität neugebildeter Phraseologismen bleibt Karlin weitestgehend in der Vorstellungswelt ihrer potentiellen Rezipienten. 
(3) Die von Karlin gebrauchten Phraseologismen wirken multifunktional. Die Sachverhaltsdarstellung, die Kommunikationssituationen und die literarischen Figuren werden durch die Phraseologismen sinnkonstituierend, argumentierend, konkretisierend, charakterisierend unterstützt. Bei der Gestaltung der Beziehung zum Adressaten besitzen die Phraseologismen eine autoritätsvertretende Funktion bezüglich des aE, sie stiften andererseits Vertraulichkeit. Generell können die Phraseologismen die denotativen Sachverhalte für Rezipienten veranschaulichen und deren Phantasie anregen, was insbesondere durch die metaphorisch unterlegten Phraseologismen geschieht. Hinsichtlich der Textbotschaft nutzt Karlin die Emotionalisierungsfunktion der isotopiebildenden Phraseologismen aus.

(4) Die Selbstdarstellung der Autorin kann an der Textbotschaft festgemacht werden, die vom aE in einem wesentlichen Textem mit phraseologischer Stützung formuliert wird. Für Rezipienten auffällig ist Karlins Einstellung zur Sprache. Sprachbewusst wählt die Autorin ugs. und geh. Phraseologismen aus, die einerseits die Kommunikationssituationen und die literarischen Figuren (mittels Sprachporträt) charakterisieren, andererseits autorintendiert vor allem mittels geh. Phraseologismen einen ,hohen“ Romaninhalt simulieren. Quantitativ auffällig ist die Neubildung ungewöhnlicher PL und satzwertiger Phraseologismen. Ihre sprachliche Kreativität stellt Karlin insbesondere in Modifikationen unter Beweis, wobei sie die Phraseologismen textuell stimmig einbettet, aber auch künstlerisch geformte Nominationen einbringt.

(5) $\mathrm{Zu}$ resümieren ist, dass sich die sprachliche Kreativität Karlins bezüglich der Phraseologismen auf verschiedenste Aspekte bezieht: z. B. auf die sprachbewusste Auswahl bestimmter Phraseologismen hinsichtlich Textbedeutung und Textbotschaft, auf die Neubildung von Phraseologismen mit semantischen Akzentuierungen, auf die kontextuale Einbettung inklusive sprachlicher Kommentierungen, auf die Bevorzugung von Modifikationsarten. Auch wenn Karlin keine Autorphraseologismen bildet, offerieren sich die okkasionellen Phraseologismen als gewichtiger, kreativer Baustein im stilistischen Bild des Romans.

\section{Literaturverzeichnis}

\section{Primärliteratur:}

Karlin, Alma M. (1933): Windlichter des Todes. Roman aus Siam. Leipzig.

\section{Sekundärliteratur:}

BUrger, Harald (2007): Phraseologie. Eine Einführung am Beispiel des Deutschen. Berlin.

BrodBECK, Karl-Heinz (2006): Neue Trends in der Kreativitätsforschung. In: Psychologie in Österreich, Nr. 4 \& 5, S. 246-253.

DuR 2011 = Dudenredaktion (Hrsg.) (2011): Duden Redewendungen. Mannheim u. a.

DUW 2001 = Dudenredaktion (Hrsg.) (2001): Deutsches Universalwörterbuch. Mannheim u. a.

EhrHARDT, Horst (2015): Personenbenennungen bei Alma M. Karlin. In: VAlENČIC ArH, Urška / Čuden, Darko (Hrsg.): V labirintu jezika. Im Labyrinth der Sprache. Ljubljana, S. 67-82.

FleISCHER, Wolfgang (1997): Phraseologie der deutschen Gegenwartssprache. Tübingen.

Handbuch 1982 = Burger, Harald / BuHOFER, Annelies / SiALM, Ambros (Hrsg.) (1982): Handbuch der Phraseologie. Berlin; New York.

Hessky, Regina / EtTINGER, Stefan (1997): Deutsche Redewendungen. Ein Wörter- und Übungsbuch für Fortgeschrittene. Tübingen.

MusolfF, Andreas (1990): Kommunikative Kreativität. Aachen.

PALM, Christine (1995): Phraseologie. Eine Einführung. Tübingen.

PoHL, Inge (2019a): Epische Darstellungstechniken in Alma M. Karlins „Windlichter des Todes. Ro- 
man aus Siam“. Eine textlinguistisch-stilistische Analyse. In: JESENŠEK, Vida / EHRHARDT, Horst (Hrsg.): Sprache und Stil im Werk von Alma M. Karlin. Maribor u. a., S. 137-174.

PoHL, Inge (2019b): Ungewöhnliche Wortbildung am Beispiel von Okkasionalismen in Alma M. Karlins Roman „Windlichter des Todes. Roman aus Siam“. In: JESENŠEK, Vida / EhrHARDT, Horst (Hrsg.): Sprache und Stil im Werk von Alma M. Karlin. Maribor u. a., S. 259-293.

PoHL, Inge (2019c): Kodierung von Emotionen in ,Windlichter des Todes. Roman aus Siam“ von Alma M. Karlin. In: Studia Germanistica. Acta Facultatis Philosophicae Universitatis Ostraviensis, Nr. 24, Ostrava, S. 31-46.

PolzIN, Carsten (1997): Kreativität in der Wortbildung - kontrastiv gesehen. In: Kreativität. Beiträge zum 12. Nachwuchskolloquium der Romanistik. Bonn, S. 269-279.

Pusch, Claus D. (1997): (Alltags-)Sprachliche Kreativität und Grammatikalisierung. In: Kreativität. Beiträge zum 12. Nachwuchskolloquium der Romanistik. Bonn, S. 281-292.

Richter-VAPAATAlo, Ulrike (2007): „, Da hatte das Pferd die Nüstern voll“: Gebrauch und Funktion von Phraseologie im Kinderbuch. Frankfurt am Main u. a.

Slibar, Neva (2006): ALMA KOLUMBUS AUF WELTREISE. Problematik und Potenzial der geobiographischen Bücher und exotischen Erzählungen der ,, Staatsbürgerin eines fremden Staates". In: Osolnik Kunc, Viktorija / Hudelua, Niko / Šetinc Salzmann, Madita (Hrsg.): Transkulturell - Transkulturno - Berlin - Ljubljana - Zabočevo. Ljubljana, S. 319-337.

SpriL 1989 = BEYER, Horst und Annelies (1989): Sprichwörterlexikon. Sprichwörter und sprichwörtliche Ausdrücke aus deutschen Sammlungen vom 16. Jahrhundert bis zur Gegenwart. Leipzig.

Stamm, Ulrike (2012): Schiffbruch und Liebestod. Literarische Phantasien vom Scheitern interkultureller Beziehungen im frühen 20. Jahrhundert. In: HoldenRIED, Michaela / WILLMS, Weertje (in Zusammenarbeit mit Hermes, Stefan) (Hrsg.): Die interkulturelle Familie. Literatur- und sozialwissenschaftliche Perspektiven. Bielefeld, S. 215-233.

Štavbar, Simona (2019): Euphemismen und Dysphemismen als ein Charakteristikum vom Alma M. Karlins Reiseberichten. In: JESENŠEK, Vida / EHRHARDT, Horst (Hrsg.): Sprache und Stil im Werk von Alma M. Karlin. Maribor u. a., S. 241-257.

StEIN, Stephan (1995): Formelhafte Sprache. Untersuchungen zu ihren pragmatischen und kognitiven Funktionen im gegenwärtigen Deutsch. Frankfurt am Main. u. a.

STUMPF, Sören (2015): Formelhafte (Ir-)regularitäten. Korpuslinguistische Befunde und sprachtheoretische Überlegungen. Frankfurt am Main. u. a.

WolfF, Patrick / EhrHARdT, Horst (2017): Zum Reflex mitteleuropäischen Denkens im Phraseologismengebrauch bei Alma M. Karlin. In: JeSENŠEK, Vida (Hrsg.): Germanistik in Maribor. Tradition und Perspektiven. Maribor u. a., S. 217-234.

Wotjak, Barbara (1992): Verbale Phraseolexeme in System und Text. Tübingen. 